Florida International University

FIU Digital Commons

FIU Electronic Theses and Dissertations

University Graduate School

$11-21-1998$

\title{
Laser cooling and trapping of argon metastable atomic beam
}

Juan Carlos Catala

Florida International University

Follow this and additional works at: https://digitalcommons.fiu.edu/etd

Part of the Physics Commons

\section{Recommended Citation}

Catala, Juan Carlos, "Laser cooling and trapping of argon metastable atomic beam" (1998). FIU Electronic Theses and Dissertations. 2083.

https://digitalcommons.fiu.edu/etd/2083

This work is brought to you for free and open access by the University Graduate School at FIU Digital Commons. It has been accepted for inclusion in FIU Electronic Theses and Dissertations by an authorized administrator of FIU Digital Commons. For more information, please contact dcc@fiu.edu. 
FLORIDA INTERNATIONAL UNIVERSITY

Miami, Florida

\title{
LASER COOLING AND TRAPPING OF ARGON METASTABLE ATOMIC BEAM
}

\author{
A thesis submitted in partial satisfaction of \\ the requirements for the degree of \\ MASTER OF SCIENCE \\ in \\ PHYSICS \\ by \\ Juan Carlos Catala
}


To Dean Arthur $W$. Herriott

College of Arts \& Sciences

This thesis, written by Juan Carlos Catala, and entitled Laser cooling and Trapping of Argon Metastable Atomic Beam, having been approved in respect to the style and intellectual content, is referred to you for judgement.

We have read this thesis and recommend that it be approved.

\author{
John W. Sheldon \\ Yifu Zhu \\ John T. Landrum \\ Kenneth A. Hardy, Major Professor
}

Date of Defense: November 21, 1997

The thesis of Juan Carlos Catala is approved.

Dean Arthur w. Herriott

College of Arts and Sciences

Dr. Ríchard L. Campbell

Dean of Graduate studies

Florida International University, 1998 
I dedicate this thesis to my parents, even though they can not be with me in this important moment of my life, they know I am doing this for them so they can be proud of me; to my lovely wife Ivon whose dedication to my person and to my future does not have limit; to my best friend, my only brother in the USA, Otto Linsuain for all his time and support; to my guardian angel king ow for his attention and care; to all people who have ever encouraged me in this project. 


\section{ACKNOWLEDGEMENTS}

I would like to give my special thanks to Dr. Kenneth A. Hardy for his tremendous help in my thesis research, his good-willed urge and encouragement. Sincere thanks should be given to the other members of the committee, Drs. John w. Sheldon, John T. Landrum and Yifu Zhu, for their helpful suggestion and criticism to this thesis. Specially I would like to thank Drs. Rudolf Fiebig, stephen Mintz and Xuewen Wang for their wonderful courses and for their willingness to be helpful at any time. I would also like to express my gratitude to my lab colleagues, Mircea Guna, Juan Sebastian Oddone, Libni Simons and Gessler Hernandez for their help, support and great time spent together. A very special thanks to the Physics Department main office staff, Ofelia Adan-Fernandez, Hiddy Doren and Robert Gutierrez for their orientation and for always being there for me, and to my body Carlos orta for having a solution to every mechanical problem we had in the lab. 
ABSTRACT OF THE THESIS

LASER COOLING AND TRAPPING OF ARGON

MetagtabLe ATOMIC BEAM

by

Juan Carlos Catala

Florida International University, 1998

Miami, Florida

professor Kenneth Hardy, Major Professor

The high velocity of free atoms associated with the thermal motion, together with the velocity distribution of atoms has imposed the ultimate limitation on the precision of ultrahigh resolution spectroscopy. A sample consisting of low velocity atoms would provide a substantial improvement in spectroscopy resolution.

To overcome the problem of thermal motion, atomic physicists have pursued two goals; first, the reduction of the thermal motion (cooling); and second, the confinement of the atoms by means of electromagnetic fields (trapping). Cooling carried sufficiently far, eliminates the motional problems, whereas trapping allows for long observation times.

In this work the laser cooling and trapping of an argon atomic beam will be discussed. The experiments involve a time-of-flight spectroscopy on metastable argon atoms. Laser deceleration or cooling of atoms is achieved 
by counter propagating a photon against an atomic beam of metastable atoms. The solution to the Doppler shift problem is achieved using spatially varying magnetic field along the beam path to Zeeman shift the atomic resonance frequency so as to keep the atoms in resonance with a fixed frequency cooling laser.

For trapping experiments a Magnetooptical trap (MOT) will be used. The MOT is formed by three pairs of counterpropagating laser beams with mutual opposite circular polarization and a frequency tuned slightly below the center of the atomic resonance and superimposed on a magnetic quadrupole field. 
II.LASER COOLING OF NEUTRAI ATOMS 6

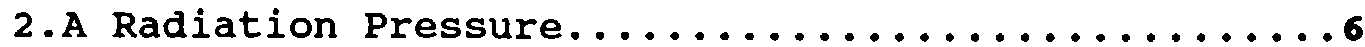

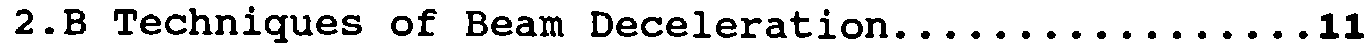

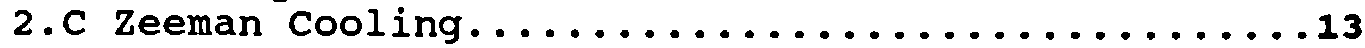

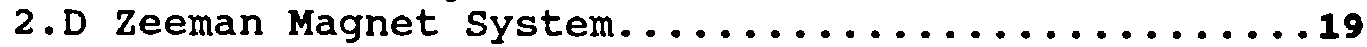

III. TRAPPING OF NEUTRAL ATOMS 25

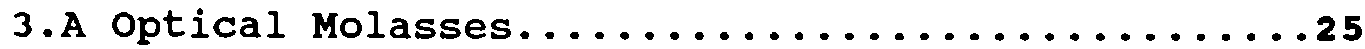

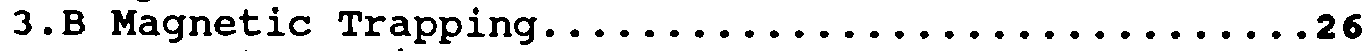

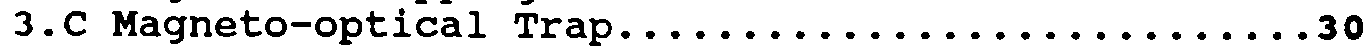

IV. RADIATION BOURCE

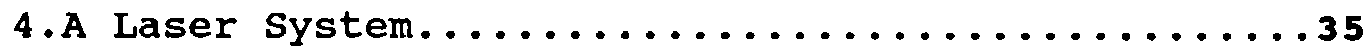

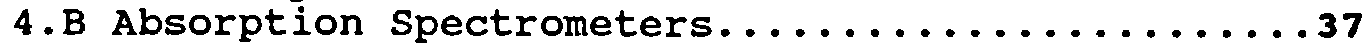

4.C Optics for laser Beam Direction.............40

V. MOLECULAR BEAM APPARATU8 43

5. A Vacuum system.........................43

5.B Source and chopper....................... 45

5.C Detector and Time of Flight Analysis.........48

5.D Data Acquisition.......................49

VI. ATOMIC COOLING EXPERIMENTS 53

6.A Hole-burning Experiments.................53

6. . Cooling Experiments.....................63

$\begin{array}{lr}\text { VII CONCLUSION } & 72\end{array}$

LIST OF REFERENCES

$\begin{array}{ll}\text { APPENDIX I } & 76\end{array}$ 
Figure 2.1 Absorption-emission Cycles.............8

Figure 2.2 Energy Level Diagram for Argon..........15

Figure 2.3 Zeeman Effect for an Atomic

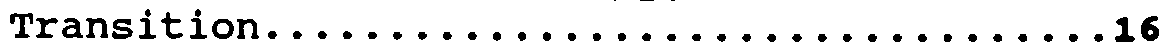

Figure 2.4 Magnetic Field Profile at Constant Currents.................21

Figure 2.5 Tapered Magnetic Field Profile.........23

Figure 3.1 Trapping Magnetic Field............28

Figure 3.2 The Equipotential of the Quadrupole Magnetic Field.............29

Figure 3.3 Arrangement for a MOT.............32

Figure 4.1 Schematic of the Optics for Cooling Experiments......................

Figure 4.2 Schematic of the Optics to Create the MOT...................12

Figure 5.1 Molecular Beam Apparatus..............4

Figure 5.2 Typical TOF Distribution............52

Figure 6.1-6.4 a)b) Hole-burning Spectra and the Absorption Curves........56-59

Figure 6.5-6.6 Hole-burning Spectra with Linearly Polarized Laser and Constant Magnetic Field.........61-62

Figure 6.7-6.9 Hole-burning Spectra with Positive Circularly Polarized Laser and Constant Magnetic Field......64-66

Figure 6.10-6.12 a)b) Laser Cooling Spectra and the Absorption Curves.......67-69 


\section{LIST OF TABLES}

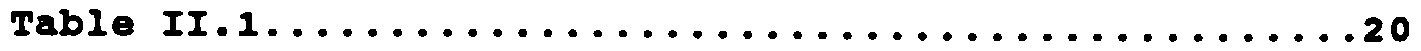

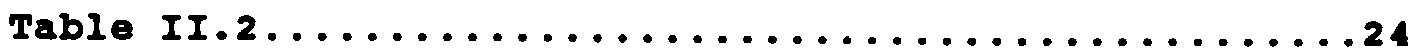

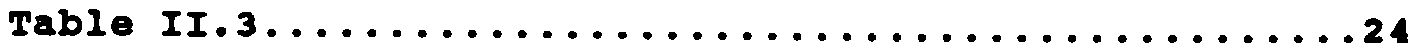

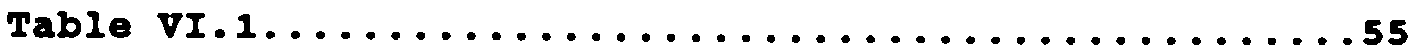

Table vr.2...........................60

Table vI.3...........................60

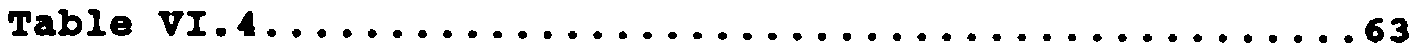

Table vr.5..........................

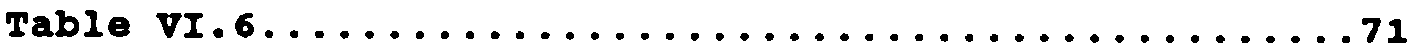




\section{Chapter I - INTRODUCTION}

One major limitation to the performance of high precision experiments in Atomic Physics is the velocity distribution of the atoms associated with the thermal motion (the most probable velocity of the thermal distribution is typically $700 \mathrm{~m} / \mathrm{sec}$ at ordinary temperature). The average kinetic energy of this motion is directly proportional to absolute temperature and thus can never be eliminated except at the unattainable temperature of absolute zero.

With lasers, it is possible to realize conditions where atomic particles are acted upon by large light pressure forces for long periods of time. This has drastically changed the attitude of investigators about the use of light pressure on atoms. Light pressure has turned out to be an effective tool for controlling the motion of atomic particles.

To overcome the problems of thermal motion, atomic physicists have pursued two goals, (I) the reduction of the thermal motion (cooling) and (II) the confinement of the atoms by means of electromagnetic fields (trapping). Cooling, if sufficient, eliminates the problems of the thermal motion. Trapping allows long observation times. Laser cooling was first demonstrated on trapped ions. Ions were trapped first and then laser-cooled. ${ }^{1-3}$ Neutral atoms must be laser cooled before they can be trapped. When an atom absorbs the energy of a photon, making a 
transition to a more energetic state, it also absorbs the momentum of the photon and this changes the atomic velocity. Laser cooling uses this velocity change to reduce the thermal motion. The choice of an atom to use in cooling experiments depends on the availability of suitable lasers and the ease of making an intense atomic beam. Also, the spontaneous fluorescence rate must be high enough to obtain deceleration from thermal to zero velocity in a reasonably sized apparatus.

The importance of confining atomic particles in a limited volume speaks for itself, since a localized particle is an ideal object for high precision experiments. The most important application of the light force in the field of atomic physics and spectroscopy is the continuous confinement of previously cooled atoms in light or magnetic traps.

One of the applications of previously laser cooled, and then magnito-optically trapped atoms is atomic ultrahigh resolution spectroscopy. The possibility of doing spectroscopy with low velocity atoms decreases the problems caused by the shift and the spread in the transition frequencies between atomic energy levels, and by the small observation time on quickly moving atoms (Doppler effects, the broadening of the spectral line $(\Delta v \Delta \tau \approx \hbar))$. There are techniques for observation of optical spectra that are nearly free of the first-order Doppler effect, ${ }^{4-9}$ but the second-order Doppler effect, associated with the 
relativistic time dilation, is still present. A sample consisting of low velocity or mono-velocity atoms provides a substantial improvement in spectroscopic resolution.

"Detailed studies of collision phenomena, which require precise knowledge of the initial velocities, are similarly hampered by the randomness of thermal motion. Neither the direction nor the magnitude of the relative velocity of two colliding atoms is well defined when velocities are distributed thermally. Velocity selection using a mechanical chopper leads to a well-defined velocity, but selection is inefficient in that most of the atoms in the atomic beam are not used. Velocity compression techniques can yield high beam flux with relative welldefined velocity, but low velocities are not attainable. ${ }^{10} \mathrm{~A}$ laser-cooled atomic beam makes efficient use of the atoms, and achieves a narrow and selectable velocity. Laser-cooling has the additional advantage of being able to place all the atoms in a single internal quantum state and at low velocities." 11

"Bose-Einstein condensation (BEC) occurs when atoms are so cold that the thermal de Broglie wavelength becomes larger than the mean spacing between atoms and their quantum nature becomes predominant. For a gas of particles with mass $m$ at temperature $T$, the de Broglie wavelength $\lambda$ equals $\mathrm{h} /(2 \pi \mathrm{mk} T)^{1 / 2}$. Although there are profound differences between fermions and bosons on the microscopic quantum level, the quantum statistics of atoms has never predicted 
any observable difference to the collective macroscopic properties of a real gas sample. The most striking difference is the prediction, originally by Einstein, ${ }^{11,12}$ that a gas of noninteracting bosonic atoms will, below a certain temperature, suddenly develop a macroscopic population in the lowest energy quantum mechanical state. For an ideal gas, this phase transition occurs when the dimensionless phase-space density $\rho=n \lambda^{3}$ exceeds 2.612 (where $\mathrm{n}$ is the number density). If the density of the gas is low (as is required for a real gas to approximate an ideal gas) this condition requires extremely low temperatures. Recently, BEC was reported in different atomic gases. ${ }^{13-15}$ Atoms were laser precooled and then confined in magnetooptical traps. A further evaporative cooling process produced record low temperatures and observation of the condensed state."11

In this work the laser cooling of an argon atom beam will be discussed. The experiments involve the time-offlight (TOF) spectroscopy of metastable argon atoms. Chapter II will explain the theory of laser cooling, especially the "Zeeman" cooling. The theory of magneto-optical trap (MOT) will be discussed in Chapter III, together with a detailed description of the optical molasses configuration, and techniques to reach the needed magnetic field gradient. The laser system used in the experiments, the absorption measurements for laser diagnostics, and the optics used to direct the laser beam will be described in Chapter IV. In 
Chapter $V$, the atomic beam apparatus is described. The laser hole-burning, the effect of constant magnetic field on the TOF-distribution, and the results of laser cooling, and trapping experiments will be discussed in Chapter VI. Finally Chapter VII will summarize the results of this investigation. 


\section{Chapter II - LASER COOLING OF NEUTRAL ATOMS}

\section{A. RADIATION PRESSURE}

The possibility of the manipulation and control of the motion of free atoms by light pressure was recognized in 1917 when Einstein discovered the momentum of light quanta $(p=\hbar k)$. Einstein stated that "if a photon strikes an atom, the atom can either absorb or emit a quanta of energy h $v$ in the form of radiation, then the momentum $h v / c$ is transferred to the atom. If it is an absorption process, the momentum transferred from the radiation source to the molecule is in the direction of the photon is coming from. For an emission process the transfer of momentum is in the opposite direction of the emitted radiation bundle."16

When a laser is directed against an incoming atomic beam, the atoms that are moving with velocity $\mathrm{V}$ will see the laser's frequency Doppler-shifted $\nu=\left(\nu_{I}-\delta\right)(1+V / C)$. To compensate for this shift the laser's frequency should be tuned to a frequency below the frequency of resonance absorption $\nu_{r}$.

If the detuning $\delta=\nu_{r} \mathrm{~V} / \mathrm{C}$, compensates for the Doppler shift corresponding to a velocity $V$, then the atom sees the laser frequency resonant $v=\nu_{r}$ and will absorb the photon. Once the photon is absorbed, the atom will store the energy by going into an excited state; to conserve the linear momentum it will recoil with a $\mathrm{h} v / \mathrm{c}$ momentum in the same direction as the laser beam; and the internal motion of 
its electrons will compensate for the photon's angular momentum $(\hbar)$.

After each absorption, the atom's velocity is reduced by $\Delta v=h v / M c$, where $M$ is the mass of the atom. This is the velocity change that is responsible for the laser deceleration or cooling (typically few $\mathrm{cm} / \mathrm{s}$, for Argon $\Delta V$ equals to $1.2 \mathrm{~cm} / \mathrm{sec}$ per absorbed photon). This rate is very small compared with an atom's thermal velocities, but it is possible to obtain low velocity atoms after multiple absorption. For example: to stop an Argon atom with velocity of $700 \mathrm{~m} / \mathrm{sec}$, over $5.8 \times 10^{4}$ photons must be scattered. (see appendix 1)

An atom in an excited state can radiate a photon by spontaneous or stimulated emission, in both cases it will recoil. In stimulated emission, photons are emitted in the same direction as the incoming laser beam. This produces a cancellation of the momentum transferred by the absorbed photon. In spontaneous emission, photons may be emitted in any direction. Therefore, the net momentum transferred due to spontaneous emission will be zero when averaged over many emissions. This makes the net momentum transfer after absorption equal to the momentum transfer of the absorption (see figure 2.1).

After the atom absorbs $\mathrm{N}$ photons, the velocity change will be equal to $\mathrm{N} \Delta \mathrm{V}$ and for the spontaneous emission 
absorption-spontaneous emission cycle

$p=h v / c \quad E=h v$

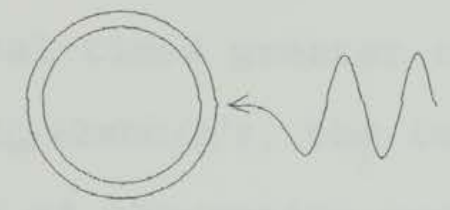

$\mathrm{M} \Delta \mathrm{V}=\mathrm{h} v / \mathrm{c}$

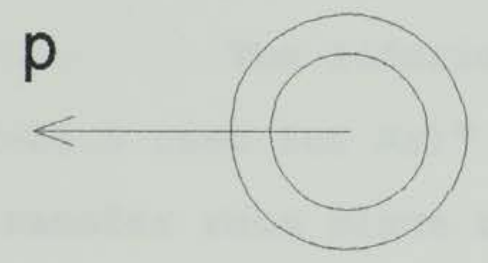

absorption-stimulated emission cycle

$$
p=h v / c \quad E=h v
$$

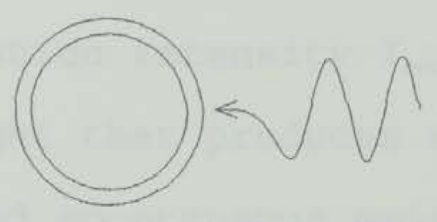

$M \Delta V=h v / c$

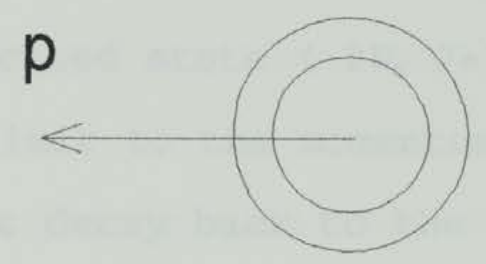

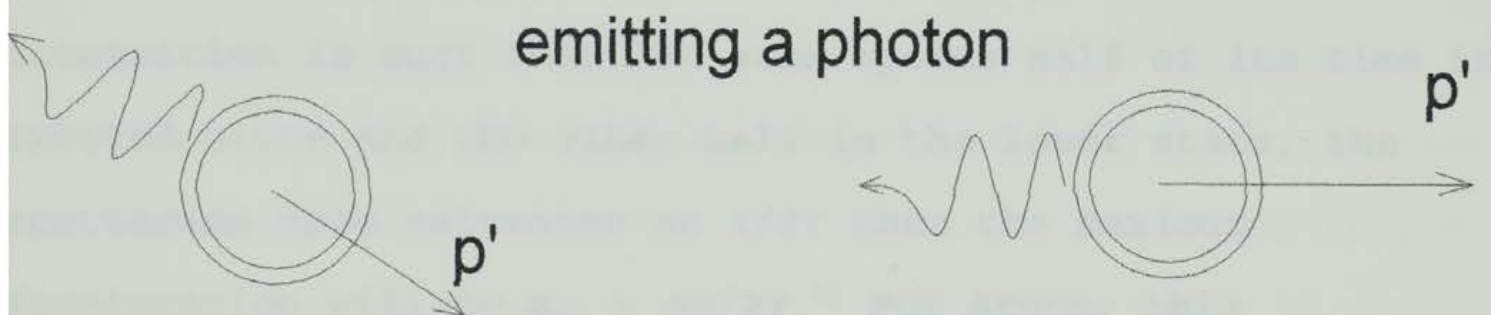

$\left\langle p_{1}^{\prime}\right\rangle=0$ after $\mathrm{N}$ cycles

$\left\langle p^{\prime}\right\rangle=-p$ after $N$ cycles

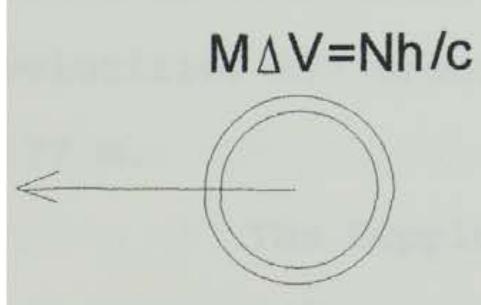

NO NET MOMENTUM TRANSFER

Figure 2.1. Absorption-emission cycles 
case the atom will receive an accumulated momentum in the direction of the laser beam and will decelerate.

The deceleration of atoms requires spontaneous absorption-emission cycle and because of this, it is limited by the spontaneous fluorescence rate. The maximum attainable deceleration is achieved at high light intensities that are several times greater than the saturation intensity $I_{\text {sat }}$ ( $I>>I_{\text {6at }}=2 \pi h c / \lambda^{3} \tau$, the intensity of light that produces equal rates of absorption and stimulated and spontaneous emission) for most of the atomic transitions. This is a few $\mathrm{mw} / \mathrm{cm}^{2}$ (for Ar atoms $I_{\mathrm{sat}}=8 \mathrm{~mW} / \mathrm{cm}^{2}$ ).

The lifetime of the excited state ( $2 \mathrm{P}_{9} \mathrm{~J}=3$ with $\tau \sim 28.5 \mathrm{nsec}$ for $\mathrm{Ar})^{21}$ imposes a limit to the momentum transfer rate since the atom must decay back to the lower energy state from which it was excited. If the light intensities is such that the atom spends half of its time in excited state and the other half in the lower state, the scattering rate saturates at $1 / 2 \tau$ then the maximum deceleration will be $\mathrm{a}_{\max }=\Delta \mathrm{v} / 2 \tau .{ }^{20}$ For Argon, this corresponds to a deceleration of about $2.16 \times 10^{5} \mathrm{~m} / \mathrm{sec}^{2}$. This would be sufficient to bring thermal Argon atoms with velocities $577 \mathrm{~m} / \mathrm{sec}$ to rest in $3 \mathrm{msec}$ over a distance of $.77 \mathrm{~m}$.

The Doppler-shifted laser frequency in the moving atom's rest frame should match that of the atomic transition to maximize the light absorption and scattering rate. This rate is given by the Lorentzian: ${ }^{17}$ 


$$
\Gamma_{s c}=\frac{\gamma s / 2}{1+S+(4 \pi)^{2} \frac{\left(\delta-\Delta \nu_{d}\right)^{2}}{\gamma^{2}}}
$$

Where $\mathbf{s}=I / I_{\mathbf{a r a}}$ is the ratio of the light intensity I to the saturation intensity $I_{\text {a at }} ; \delta=\nu_{\mathrm{r}}-\nu_{1}$ is the detuning of the laser frequency $\nu_{1}$ from the atomic resonance frequency $\nu_{r} ; \quad \Delta v_{d}=v_{r} V / C$ is the Doppler shift corresponding to velocity $\mathrm{V}$; and $\mathrm{Y}=1 / \tau$.

For maximum deceleration, $\delta-\Delta \nu_{d}$ must be chosen to be nearly zero so that the laser is in resonance with the atom having a velocity $\mathrm{V}$ in the atom's rest frame. The net force on an atom will be $\mathrm{F}=\mathrm{h} \nu_{\mathrm{x}} \Gamma_{\mathrm{sc}} / \mathrm{C}$ and becomes, at large $\mathbf{s}$, $\mathrm{h} \nu_{\mathrm{r}} / 2 \tau$.

The scattering process is stopped by the Doppler shift, making possible the absorption of just a few hundred of photons before the Doppler shift will take them out of resonance with the spectrally narrow laser. This terminates the cooling process. A velocity change of a few meters per second will correspond to a Doppler shift decrease of a few times the natural linewidth of the optical transition (typically few MHz). As an example: a velocity change of 8 $\mathrm{m} / \mathrm{sec}$ will correspond to a decrease in the Doppler shift of 2 times the natural linewidth of the optical transition in Argon (5.6 MHz for a cycling transition that is used in this experiment, see appendix 1). As a result the only atoms that see the laser frequency in resonance are slightly decelerated by a few meters per second before they go out of 
resonance with the laser. This phenomena will produce a very narrow hole in the velocity distribution. This comes about as those atoms that were initially nearly resonant with the laser are decelerated to lower velocities where they accumulate as they go out of resonance and no longer experience any significant changes in their velocities. This is called hole burning in the velocity distribution curve and it is a clear experimental evidence of laser cooling of an atomic beam. The hole burned in the TOF distribution can be approximate to a Lorentzian.

\section{B. TECHNIQUES OF BEAM DECELERATION}

The problem of cooling neutral atoms in an atomic beam is to avoid the limitations imposed by the changing Doppler shift which takes the atoms out of resonance with the radiation light source. A solution to this problem is needed in order to cool a large number of atoms.

The first solution for such a problem was chirp cooling and was suggested by Lethokov ${ }^{18}$. Chirp cooling consists of varying the laser's frequency as the atoms are decelerated. The sweeping (or chirping) of the laser frequency from $v$ to $v+\Delta v$ will keep the laser's frequency resonant with the Doppler-shifted decelerating atoms. All atoms with slower velocities than the initially resonant velocity $V_{i}$ are swept into a narrow velocity group around $V_{f}$ $\left(\mathrm{V}_{\mathrm{i}}-\mathrm{V}_{\mathrm{f}}=\lambda \Delta \nu\right)$. There is a maximum deceleration $\left(\mathrm{a}_{\max }\right)$ and because of this, there is also a maximum frequency scan rate 
$v_{\max }=\left(a_{\max } / \lambda\right)^{1 / 2}$. If the frequency scan rate $\nu$ of the laser is larger than $v_{\max }$, the atom's Doppler shift cannot change fast enough and the atom will go out of resonance. If the scan rate is lower than $v_{\max }$, and there is sufficient laser power, the atomic velocity will adjust itself to be just far enough out of resonance so that the scattering rate produces for the required rate of change in the Doppler shift. This technique is commonly used with diode lasers. The diode laser's frequency is changed by changing the current. Unfortunately, the implementation of this method is not as simple as it sounds. It a very difficult task to keep the frequency of the laser tuned while scanning at the necessary rate.

Another approach to the Doppler shift problem was suggested by $\operatorname{Prodan}^{19}$ and it consists of changing the frequency of the atomic transition rather than that of the laser so that there is also a constant deceleration. This method is achieved by an inhomogeneous magnetic field along the beam's path, in this case the magnetic field will shift the atomic resonance frequency (Zeeman effect) in order to keep the atoms in resonance with a fixed-frequency cooling laser.

Both techniques compress the velocity distribution and only the part of the velocity distribution that is compressed should be called "cooled", but often this term is used to include deceleration as well.

Both methods of cooling atoms addresses the same 
problem and ultimately achieve the goal. With the chirping method the compression takes place in time so all affected atoms are instantaneously in resonance with the changing laser frequency. In the zeeman shift method, the compression happens in space so all affected atoms at a particular point in space are resonant. As a result of this all the atoms are brought to rest (or to some final velocity) at the same location in the magnetic system. Another difference is that "Zeeman cooling" is a continuous process, whereas chirp cooling produces pulses of cooled atoms at the end of each laser frequency scan. Zeeman cooling produces all of the cooled atoms in the same quantum state, and this is extremely advantageous for trapping or collision experiments .

When the laser's power is greater than required by the magnetic field gradient or the chirp rate, the atomic velocity will adjust itself to be slower than the resonant velocity, so atoms faster than the average velocity, being closer to the resonance, decelerate more, while slower atoms decelerate less. This effect further compresses the velocity distribution.

Because of the simple implementation and because the final product of cooled atoms in the same quantum state, we decided to perform the experiment using Zeeman cooling.

\section{ZEEMAN COOLING}

The Zeeman cooling is possible because any atom 
under the influence of an external magnetic field will experience a shift in its energy levels; this is known as Zeeman effect.

In a magnetic field, the energy levels of an atom split according to the projection, $m_{j}$ of the total angular momentum, onto the magnetic field direction. The quantization axis is defined along the axis of the atomic and laser beams. The laser light is circularly polarized and carries angular momentum. The direction of polarization is chosen to be $\sigma^{+}$, so that the atoms absorbing the light must increase the projection of their angular momentum $\left(\Delta m_{j}=+1\right)$.

Argon has a cycling transition at $811.73 \mathrm{~nm}$ (cycles of excitation and decay involve only two states). The lower state is a $1 S_{5} \mathrm{~J}=2$ metastable state and acts as a ground state with a lifetime greater than 1.3 seconds; the upper excited state is a $2 \mathrm{P}_{9} \mathrm{~J}=3$ state with lifetime $\tau \sim 28.5$ ns. ${ }^{21}$ The natural linewidth of the transition $Y=1 / 2 \pi \tau$ is about $5.6 \mathrm{MHz}$ (see figure 2.2).

For Argon atoms in the $J=2 \quad m_{j}=2$ sublevel of the $1 S_{5}$ metastable state, the only state to which they can be excited is then $\mathrm{J}=3 \mathrm{~m}_{j}=3$ sublevel of the $2 \mathrm{P}_{9}$ excited state. The other possible $\sigma^{+}$transitions are shown in figure 2.3 . For $\sigma^{+}$or $\sigma^{-}$the selection rules for transitions between $m_{j}$ components of different electronic states $\left(\Delta m_{j}= \pm 1,0\right)$ guarantee that after many cycles of absorption-emission, all the atoms, regardless of their initial $m_{j}$, eventually end up in the states with highest projection of angular momentum, 


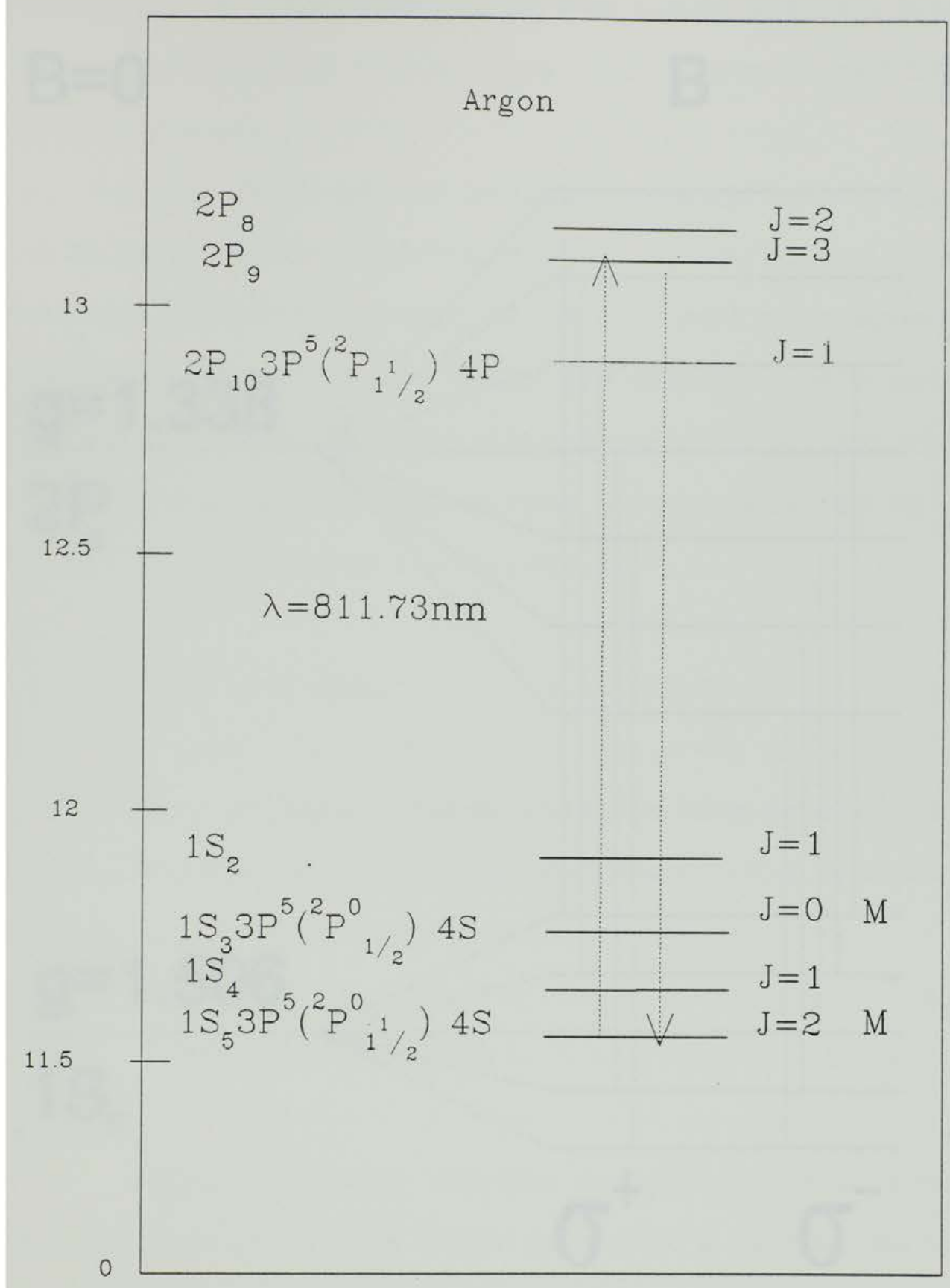

Figure 2.2 Energy Level Diagram for Argon.

The cycling transition is from the $1 S_{5} \mathrm{~J}=2$ metastable state to the $2 \mathrm{P}_{9} \mathrm{~J}=3$ state 


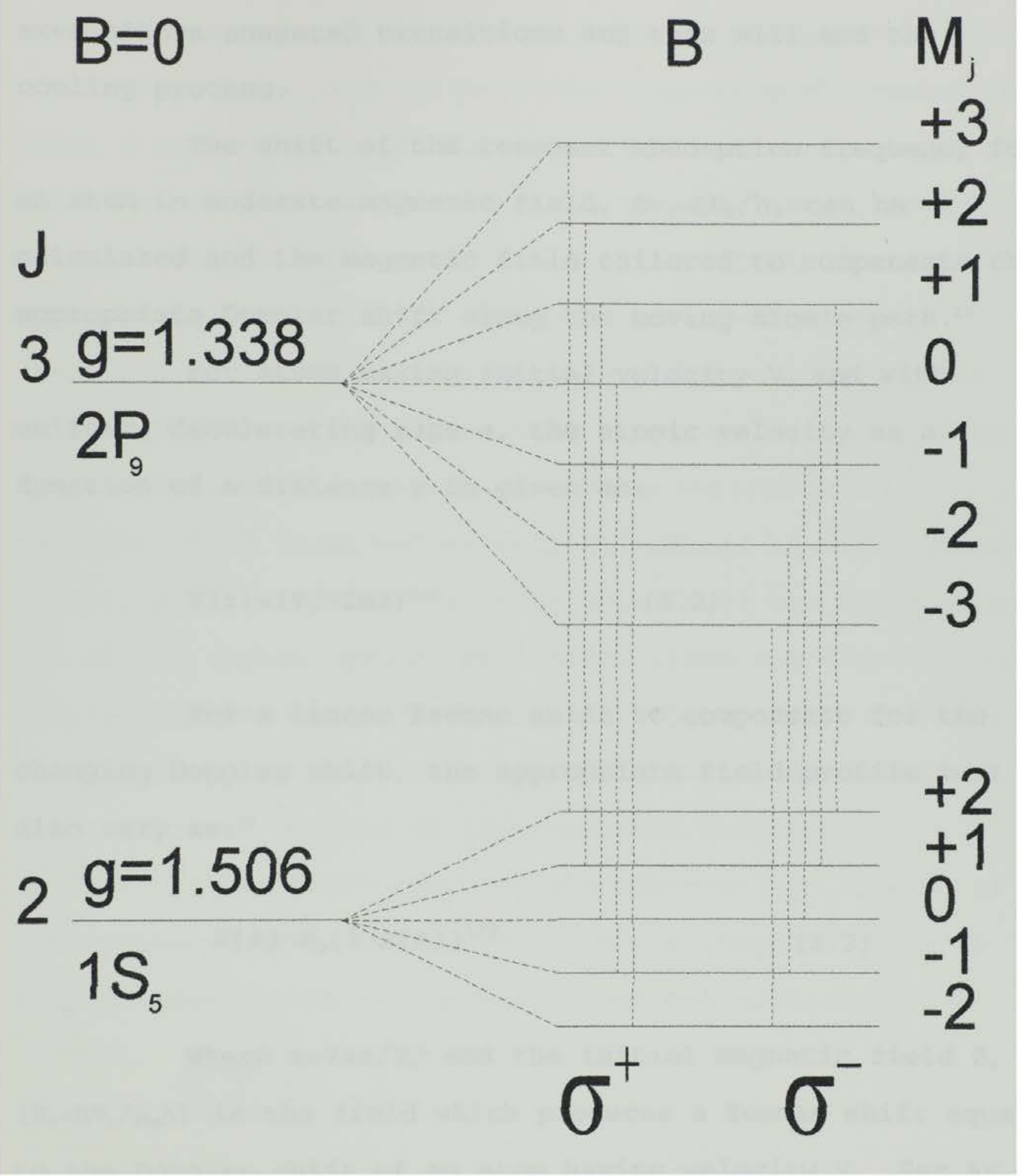

Figure 2.3. Zeeman effect for an atomic transition $\left(1 \mathrm{~S}_{5} \mathrm{~J}=2\right.$ to $1 \mathrm{P}_{9} \mathrm{~J}=3$ ) 
cycling on the $\mathrm{J}=2 \mathrm{~m}_{\mathrm{j}}=2$ to $\mathrm{J}=3 \mathrm{~m}_{\mathrm{j}}=3$ transition. Imperfect polarization or misalignment of the laser and magnetic field axes allows unwanted transitions and this will end the cooling process.

The shift of the resonant absorption frequency for an atom in moderate magnetic field, $\Delta \nu_{z}=\Delta E_{z} / h$, can be calculated and the magnetic field tailored to compensate the appropriate Doppler shift along the moving atom's path. ${ }^{19}$

For atoms having initial velocity $v_{\circ}$ and with uniform decelerating rate $a$, the atomic velocity as a function of a distance $z$ is given as:

$$
\mathrm{V}(\mathrm{z})=\left(\mathrm{V}_{0}^{2}-2 \mathrm{az}\right)^{1 / 2}
$$

For a linear Zeeman shift to compensate for the changing Doppler shift, the appropriate field profile must also vary as: ${ }^{20}$

$$
B(z)=B_{0}(1-X(z))^{1 / 2}
$$

Where $\mathrm{x}=2 \mathrm{az} / \mathrm{V}_{0}^{2}$ and the initial magnetic field $B_{\circ}$ $\left(\mathrm{B}_{\mathrm{o}}=\mathrm{hV} \mathrm{V}_{\mathrm{o}} / \mu_{\mathrm{B}} \lambda\right)$ is the field which produces a Zeeman shift equal to the Doppler shift of an atom having velocity $V_{0}$. For Ar atoms $V_{0}=577 \mathrm{~m} / \mathrm{s}$, giving a $B_{0}=509$ gauss.

There are some atoms in the thermal velocity distribution that are moving too fast to be decelerated, those will see the laser's frequency Doppler shifted too far 
into the blue to absorb light (for Ar atoms with velocities greater than $V_{0}$ ), even where the magnetic field is strongest at the magnet system entrance. Others, which have velocities equal to or less than $V_{0}$, and whose Doppler shift causes the laser frequency to match the magnetic-field shift, begin to slow down as soon as they enter the system. Atoms, that are moving so slowly that they do not absorb light, travel to a point where the spatially varying magnetic field has decreased to the value that matches their smaller Doppler shift and produce resonance. Thus all atoms with velocity $\mathrm{V}_{\mathrm{o}}$ or lower can be decelerated to some smaller final velocity. This final velocity is determined by the resonance condition at the laser frequency chosen and the field at the end of the magnet system. All cooled atoms are swept into a narrow velocity group around this final velocity. The result is that the originally wide thermal velocity distribution is compressed and shifted to lower or zero velocity.

Other field profiles are allowed, but because of the existence of a maximum possible acceleration there is also an upper limit on the field gradient given by: ${ }^{20}$

$$
\frac{d v}{d B} \frac{d B}{d z} \cdot v \leq a_{\max } / \lambda
$$

For Ar atoms $a_{\max } / \lambda=2.66 \times 10^{11} \mathrm{sec}^{-2}$.

Where $d v / d B$ depends on the Zeeman effect. This restriction is equivalent to the restriction on the scan rate of the laser in chirp cooling. 
For transition between $1 \mathrm{~S}_{5} \mathrm{~J}=2 \mathrm{~m}_{\mathrm{j}}=2$ and $2 \mathrm{P}_{9} \mathrm{~J}=3$

$m_{j}=3$ states in argon, the energy shift produced by a magnetic field of magnitude $B$ (measured in Tesla) will be $\Delta E_{z}=\left(3 g_{13}-2 g_{12}\right) \mu_{B} B$, where $\mu_{B}$ is the Bohr magneton and $g_{1}$ is the Landé $g$-factor of each state. Using $\mathrm{g}_{12}=1.506$ and $\mathrm{g}_{13}=1.338$, $\Delta \mathrm{E}_{\mathrm{z}} \approx \mu_{\mathrm{B}} \mathrm{B}$ eV and the corresponding frequency shift $\Delta \nu_{\mathrm{z}} \approx 1.4 \times 10^{10} \mathrm{~B}$ (measured in $\mathrm{Hz}$ ) or $\Delta v_{z} \approx 14 \mathrm{GHz} / \mathrm{Tesla}=1.4 \mathrm{MHz} / \mathrm{G}$. In this case, the condition given in Eqn. 2.4 can be evaluated as follow: ${ }^{11}$

$$
\mathrm{dB} / \mathrm{dz} \leq 1760 / \mathrm{V} \quad \text { [Gauss } / \mathrm{cm}]
$$

\section{MAGNET SYSTEM}

To approximate the field profile given by Eqn. 2.3 , seven solenoids $10.5 \mathrm{~cm}$ long with magnet constants of about 350 Gauss/A were constructed. ( The magnet constants measured in Gauss $/ \mathrm{A}$ are $\mathrm{M} 1=350, \mathrm{M} 2=\mathrm{M} 3=348, \mathrm{M} 4=344, \mathrm{M} 5=\mathrm{M} 6=$ 346, M7=343, respectively. These magnet constants were measured with a Gaussmeter, the measurements were done at the center of the solenoids, looking for the maximum magnetic field using the axial probe of the measuring device). The tapered magnetic field is created by using different currents, in decreasing order through each solenoid. The magnet system has a total length of $77 \mathrm{~cm}$. If the initial magnetic field, $B_{0}$, is 508 Gauss, the field will follow the theoretical profile given by Eqn. 3 with the laser tuned to the resonant frequency (detuning $\delta=0$ ). Table II.1 shows the initial magnetic field $B_{\circ}$ 
necessary to stop atoms with different initial velocities, $\mathrm{V}_{0}$, and with the corresponding length when the detuning $\delta=0$. Because of the maximum attainable deceleration, it is not possible to stop atoms with velocity greater than $577 \mathrm{~m} / \mathrm{s}$ because of the limitation imposed by magnet system length $(.77 \mathrm{~m})$

\begin{tabular}{|l|l|l|l|l|}
\hline $\mathrm{V}_{0}(\mathrm{~m} / \mathrm{s})$ & 700 & 550 & 300 & 150 \\
\hline $\mathrm{B}_{0}(\mathrm{G})$ & 617 & 485 & 264 & 132 \\
\hline $\mathrm{L}_{\mathrm{s}}(\mathrm{m})$ & 1.13 & 0.70 & 0.21 & 0.05 \\
\hline
\end{tabular}

Table II.1. $V_{0}$ is the initial velocity of the

atoms and $B_{\circ}$ is the necessary initial magnetic field to stop the atoms with $V_{0}$, and $L_{s}$ is the stopping distance.

By adjusting the current in each magnet, a magnetic field very close to the theoretical requirement can be obtained. However, because of the gap between each pair of magnets, the actual tapered magnetic field does not have a uniformly decreasing gradient. Figure 2.4 shows the measured magnetic field for two different currents, .99A and .3A respectively. In this figure is easy to see the edge effect, the current through the magnets is such that the magnetic field should be uniform all along the magnet system. The edge effect introduces a relative error at most of the $10 \%$ for currents about $1 \mathrm{~A}$; this defect decreases as the magnetic field is lowered.

A comparison between the theoretical and the measured tapered magnetic fields for two different sets of currents 


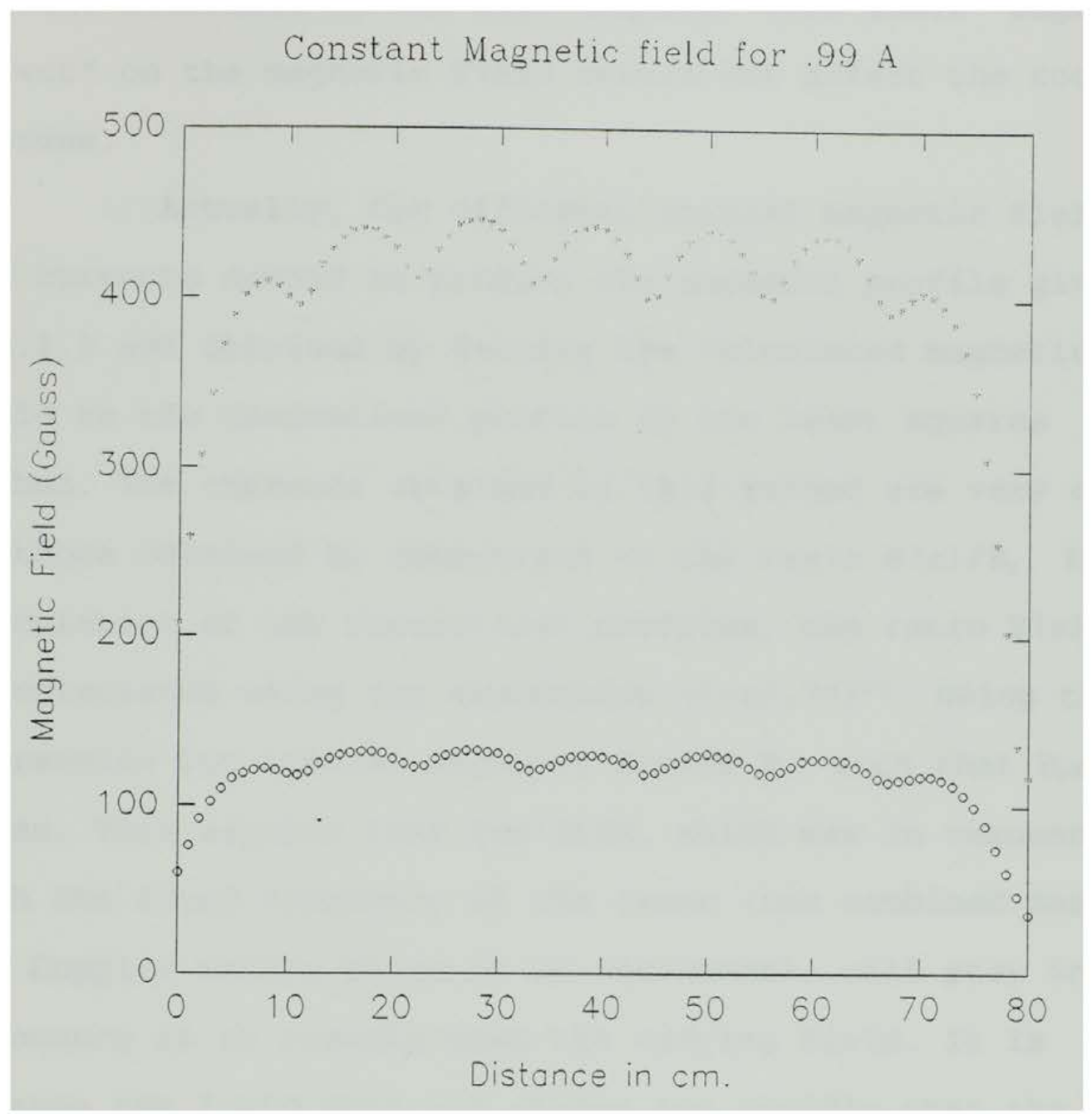

Fig. 2.4. Triangles show the measured magnetic field (MF) with .99A. in all the magnets Circles show the measured MF with .3A in all the magnets. 
is shown in figure 2.5 ( $B_{0}=490$ Gauss $)$. At the first gap, the magnetic field is about $6 \%$ different from the expected magnetic field but the difference gets smaller as the current decreases in the last magnets. This small "edge effect" on the magnetic field should not affect the cooling process.

Actually, for different initial magnetic fields, the currents needed to produce the magnetic profile given by Eqn.2.3 are obtained by fitting the calculated magnetic field to the theoretical profile by the least squares method. The currents obtained by this method are very close to those obtained by comparison to the ratio $\mathrm{B}(z) / \mathrm{B}_{0}$. In calculation of the theoretical profiles, the ratio $\mathrm{B}(z) / \mathrm{B}_{0}$ is calculated using the expression $(1-z / .77)^{1 / 2}$. Using this expression for initial magnetic fields $B_{0}$, such that $B_{0} \leq 508$ Gauss. This assures that the atom, which was in resonance with the fixed frequency of the laser (the combined zeeman and Doppler shifts place it in resonance), will stay in resonance as it travels down the varying field. It is because the field does not change too rapidly that the rate of Zeeman shift change exceeds the possible rate of Doppler shift change.

In Table II.2 the calculated currents needed to produce a tapered magnetic field with $B_{\circ}=490 \mathrm{G}$ are given. (calculated by the fitting process). 


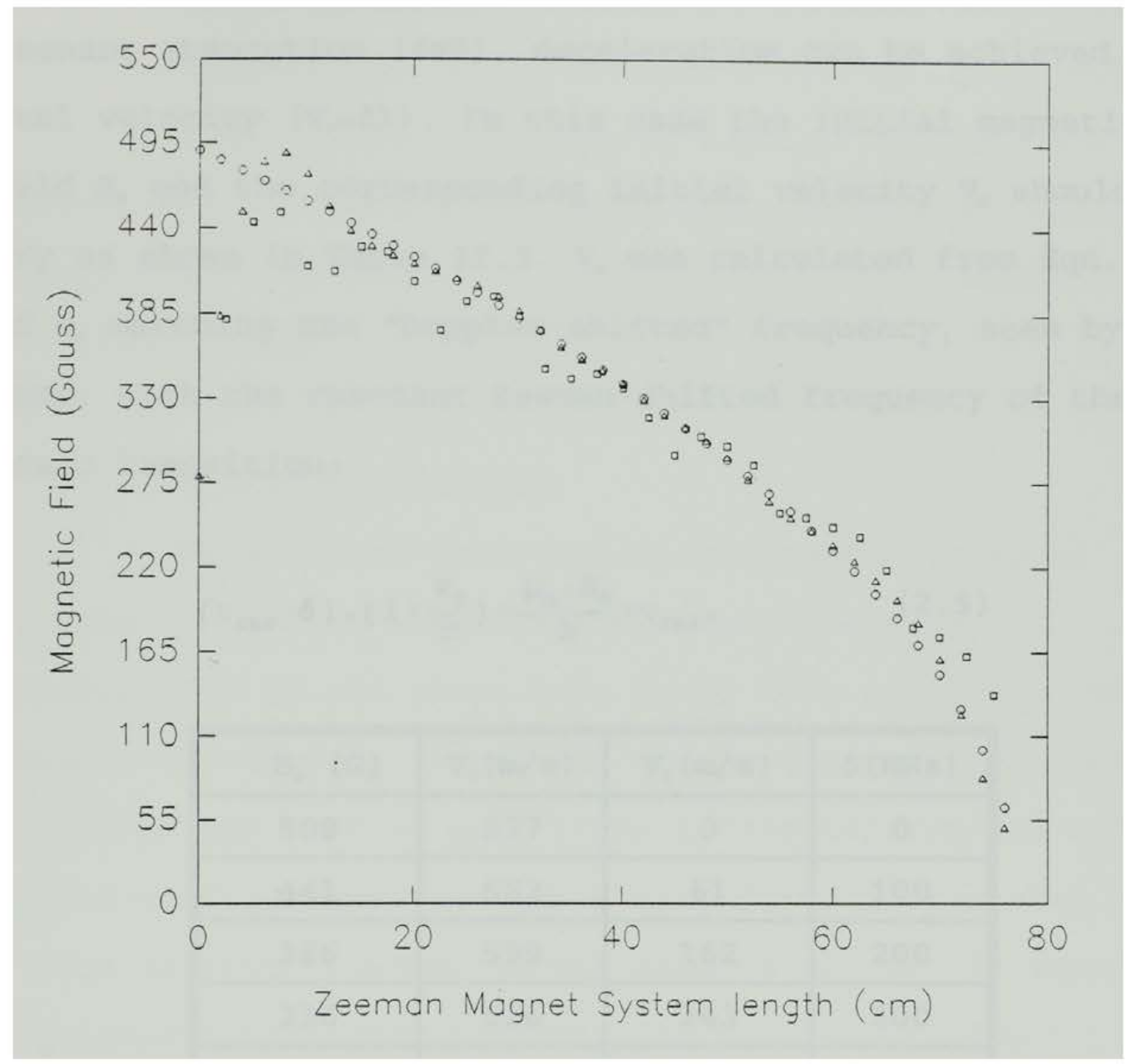

Fig 2.5 Magnetic Profiles:

circles show the theoretical MF for Bo $=490 \mathrm{G}$ trangles show the calcu;ated MF with currents: $1.2,1.03, .92, .78, .7, .55, .35$ all in amps squares show the measured MF. 


\begin{tabular}{|l|c|c|c|c|c|c|c|}
\hline Magnet & 1 & 2 & 3 & 4 & 5 & 6 & 7 \\
\hline$I_{\text {cal }}(\mathrm{A})$ & 1.3 & .93 & .86 & .76 & .69 & .53 & .47 \\
\hline
\end{tabular}

Table II.2. $I_{\text {cal }}$ is the set of currents obtained by

the fitting process.

If the laser is tuned below the frequency of resonant absorption $(\delta \neq 0)$, deceleration can be achieved the final velocity $\left(V_{f}=\delta \lambda\right)$. In this case the initial magnetic field $B_{0}$ and the corresponding initial velocity $V_{0}$ should vary as shown in Table II.3. Vo was calculated from Eqn. 2.2 and $B_{0}$ matching the "Doppler-shifted" frequency, seen by the atoms, with the resonant zeeman-shifted frequency of the atomic transition:

$$
\left(\nu_{\text {res }}-\delta\right) \cdot\left(1+\frac{v_{0}}{C}\right)=\frac{\mu_{B} \cdot B_{0}}{h}+\nu_{r e s} \cdot
$$

\begin{tabular}{||c|c|c|c||}
\hline$B_{\circ}(G)$ & $V_{o}(m / s)$ & $V_{f}(m / s)$ & $\delta(M H z)$ \\
\hline 508 & 577 & 0 & 0 \\
\hline 441 & 582 & 81 & 100 \\
\hline 386 & 599 & 162 & 200 \\
\hline 336 & 626 & 243 & 300 \\
\hline 300 & 662 & 325 & 400 \\
\hline 265 & 705 & 406 & 500 \\
\hline
\end{tabular}

Table II.3. $\delta$ is the laser detuning; $\bar{V}_{f}$ is the

final velocity corresponding to the detuning $\delta, V_{\circ}$ is the initial velocity that may have the atoms to decelerate to $V_{f}$ if the initial magnetic field is $B_{0}$. 


\section{CHAPTER III TRAPPING OF NEUTRAL ATOMS}

The ultimate goal of this experiment is to trap precooled Argon atoms. In order to trap any object it is necessary to exchange kinetic for potential energy. Since the strong coulomb interaction does not exist for neutral particles, a magneto-optical trap (MOT) can be used to create the trapping fields. The MOT is a combination of a optical molasses (three pairs of counterpropagating laser beams) and a magnetic trap (a magnetic quadrupole field).

\section{A.OPTICAL MOLASSES}

Using three intersecting orthogonal pairs of oppositely directed low intensity laser beams (by retroflection of the three main beams from a mirror, the movement of atoms is restricted to the intersecting region, collecting and cooling many atoms in the illuminated volume. In this configuration the scattering force is canceled for an atom at rest, however if the atom is moving, it will experience a net force proportional to its velocity.

The laser frequency determines the sign of the force, if the laser is tuned below atomic resonance the light in the beam opposing the atom's motion will be Doppler shifted to the blue in the atom's rest frame, the light in the beam parallel to the atom's motion will be shifted to the red. In the first case the atom will be closer to the resonance, and in the second the atom is further out of it. 
For the low intensity laser beams, low enough so that stimulated emission is unimportant, the resulting scattering force in the molasses can be calculated by adding the negative and the positive force $\left(F_{t}= \pm\left(\hbar k \gamma s / 2\left(1+S+\Delta_{ \pm}\right)\right)\right):{ }^{19}$

$$
F_{O M}=8 \hbar k^{2} \delta \mathrm{sv} / \mathrm{Y}(\mathrm{L}+\mathrm{S})^{2}
$$

Where $\delta$ is the detuning, $\mathrm{k}$ is the wave vector, $L=1+\Delta^{2}, \quad \Delta_{t}=\Delta-\nu_{D}, \Delta=2 \delta / Y$, and $\nu_{D}$ is the Doppler shift, $s$ is the ratio of the laser intensity to the saturation intensity $\left(S=I / I_{\text {sat }}\right)$, and $Y$ is the natural linewidth. This force will oppose the velocity if $\delta$ is negative and therefore viscously damps the atomic motion.

The final temperature in an optical molasses, (the Doppler cooling limit $T_{D}$ ) can be calculated from the kinetic energy for $\delta=-\gamma / 2$, and is equal to:

$$
\mathrm{T}_{\mathrm{D}}=\hbar \mathrm{\gamma} / 2 \mathrm{k}_{\mathrm{B}}
$$

Where $\mathrm{k}_{\mathrm{B}}$ stands for the Boltzmann's constant. For the case of Argon transitions at $\lambda=811.73 \mathrm{~nm} \quad \mathrm{~T}_{\mathrm{D}}=19.42 \mu \mathrm{K}$.

Although, the confiment times of atoms caught in OM can be long, it is not a trap because there is not a restoring force on an atom that has been displaced from the center.

\section{B.MAGNETIC TRAPPING}

An atom can be confined in an inhomogeneous magnetic field because this field will interact with an atom's magnetic moment. This interaction produces a force 
proportional to the gradient of the scalar product between the magnetic field and the magnetic moment of the atoms $(\mathbf{F}=\nabla(\mu \bullet \mathrm{B}))$.

Because of the angular dependence of this force, the orientation of the magnetic moment with respect to the field must be preserved as the atom moves in the trap. This condition requires low velocities, such that, the interaction between the moment and the field is adiabatic. The simplest possible magnetic trap can be constructed out of two separated coils carrying opposite currents, this is shown in figure 3.1. This will be a quadrupole trap with a single center, where the magnetic field is zero. The equipotential lines produced by this field are shown in figure 3.2. This kind of trap will have equal depth in the radial ( $\mathrm{x}-\mathrm{y}$ plane) and longitudinal ( $\mathrm{z}$ axis) directions, if the coils are separated by 1.25 times their radius. For this configuration of trap the field magnitude is equal to zero at the center and increases in all direction according to:

$$
|B|=A\left(p^{2}+4 z^{2}\right)^{1 / 2}
$$

where $\rho^{2}=x^{2}+y^{2}$ and $A$ is a constant. The field gradient will be constant along any line through the origin, but has different values in different polar directions, and because of this the trapping force is neither harmonic nor central, and the angular momentum is not conserved. ${ }^{19}$ For trapping with this quadrupole field the orbital angular momentum of the trapped atom has to allow 


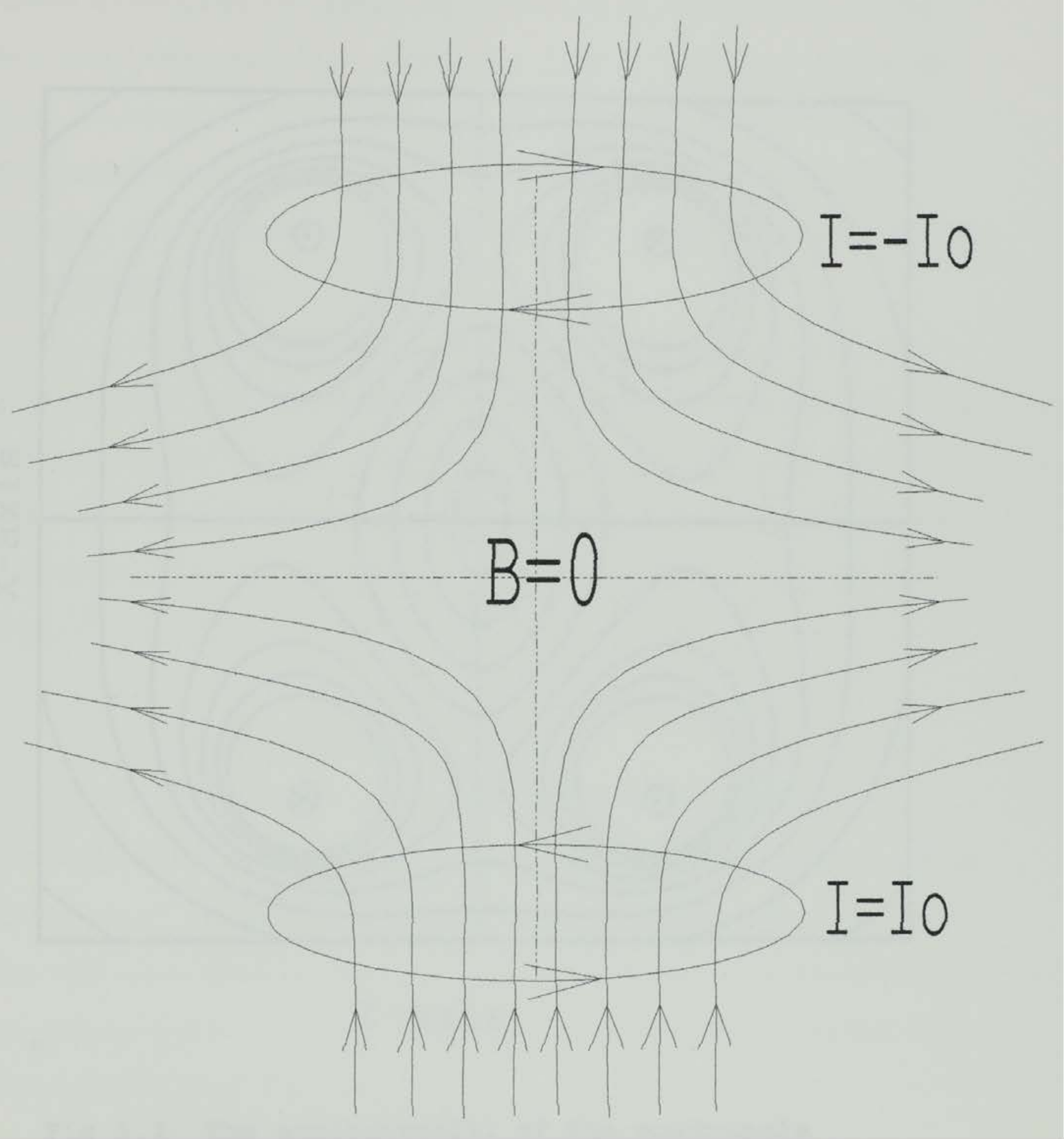

Fig 3.2. Trapping magnetic field produced by two coils with opposite running currents 


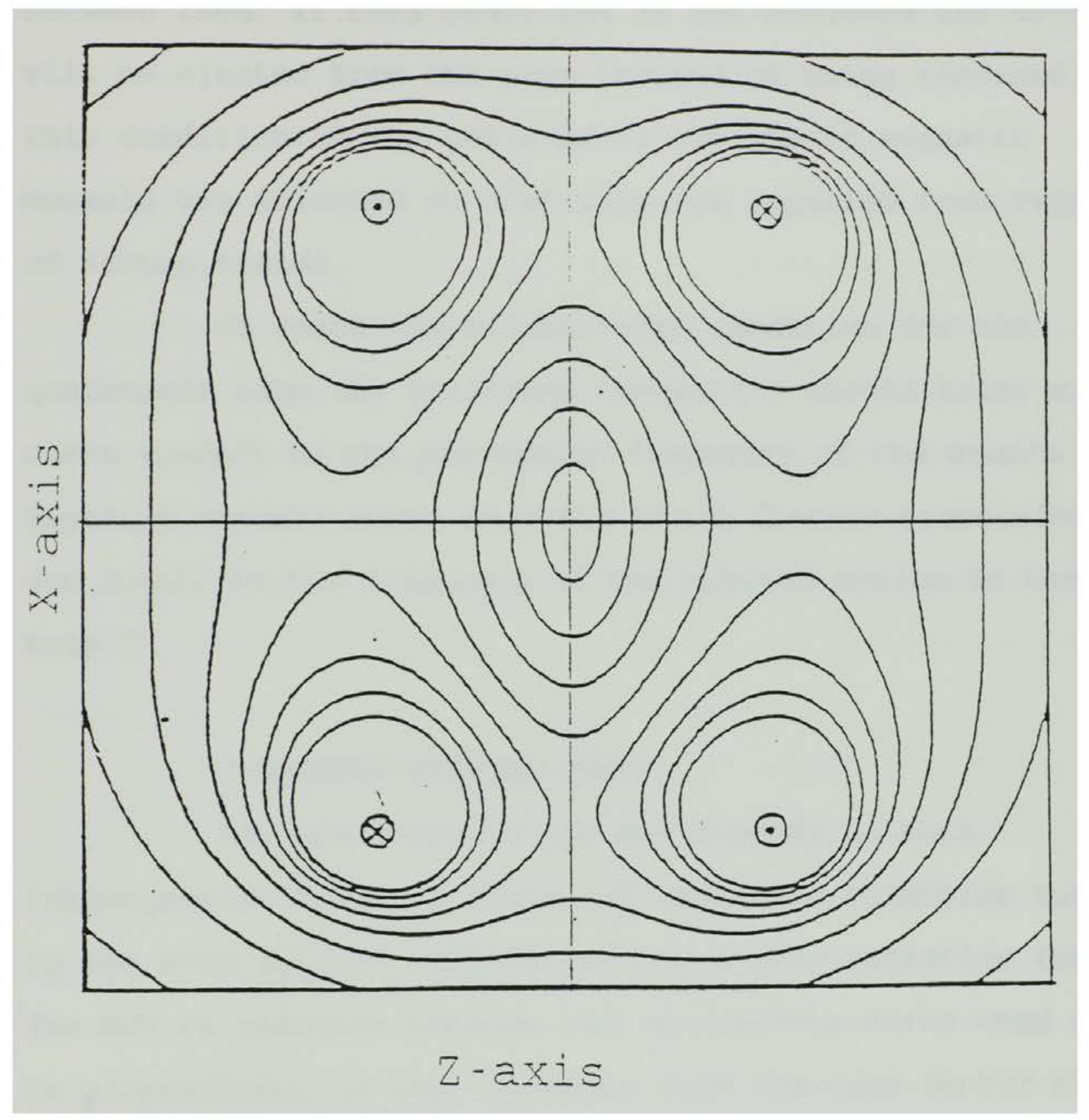

Fig 3.2. The equipotential of the quadrupole magnetic trapping field 
the orientation of its dipole moment to follow the changing field direction to conserve the sign of the interaction between them. If this condition is not achieved the atom will be ejected from the trap instead of being confined. This condition can be achieved if the atomic magnetic moments are oriented so that they are repelled from regions of strong fields.

To reach the adiabaticity condition for the quadrupole trap the following inequality should hold: $w_{\mathrm{L}} \gg \mathrm{w}_{\mathrm{T}}$ where $\mathrm{w}_{\mathrm{L}}=\mu \mathrm{B} / \hbar$ is the precession frequency of the atom's magnetic moments about magnetic field (Larmor precession) and $\mathrm{w}_{\mathrm{T}}=\mathrm{V} / \mathrm{p}$ is the frequency of the orbital motion in the trap. ${ }^{19}$

\section{C.MAGNETO-OPTICAL TRAP}

The operation of the MOT depends on both inhomogeneous magnetic field and radiative selection rules to use both optical pumping and the strong radiative force. The MOT is possible because the spontaneous force need not be proportional to the intensity with the same factor of proportionality for the entire trapping region. ${ }^{20}$

The standard configuration of the MOT was suggested by Dalibard for one dimension. ${ }^{21}$ The 3-dimension MOT is formed out of three pairs of counter propagating laser beams with mutual opposite circular polarization and a frequency tuned slightly below the center of the atomic resonance with a magnetic quadrupole field superimposing 
them.

The basic principle of confiment in a MOT can be explained using a simplified one dimension configuration shown in figure $3.3 .{ }^{22}$ The Zeeman sublevels of an atom at a distance $z$ from trap center are shifted by the local magnetic field in such a way that the selection rules cause the atom to be in resonance with the positive circular polarized $\left(\sigma^{+}\right)$laser beam at $z=-z^{*}$, and with the negative circular polarized laser beam at $z=+z^{*}$, so an atom at $+z^{*}$ is driven toward $-z^{*}$ and viceversa, this way the atom bounces back and forth between this two turning points, which can be change by tuning the laser or changing the magnetic field strength. This resonance condition is for the atom's rest frame where there is no Doppler effect.

When the Doppler effect is taken into account, it shift the optical frequencies in the atomic rest frame, so there are two different frequenciesof the counterpropagating lasers according to their polarization. For an atom moving to the right the $\sigma^{-}$beam is shifted much closer to the resonance $\left(\Delta^{-}\right)$and the $\sigma^{+}$beam much farther from resonance $\left(\Delta^{+}\right)$. Combining the Zeeman and Doppler shift the resonance condition for both type of polarization is given by: ${ }^{22}$

$$
\delta= \pm\left(2 \pi \mathrm{V} / \lambda+\mu^{\prime} \mathrm{B} / \hbar\right)
$$

Where $\mathrm{V}$ is the atom velocity, $\lambda$ is the transition wavelength, $\mu^{\prime}$ is the Zeeman tuning coefficient, $B$ is the trapping magnetic field, and $\hbar$ is the Planck's constant over $2 \pi$. A fixed detuning $\delta$ produces resonance for one beam and 


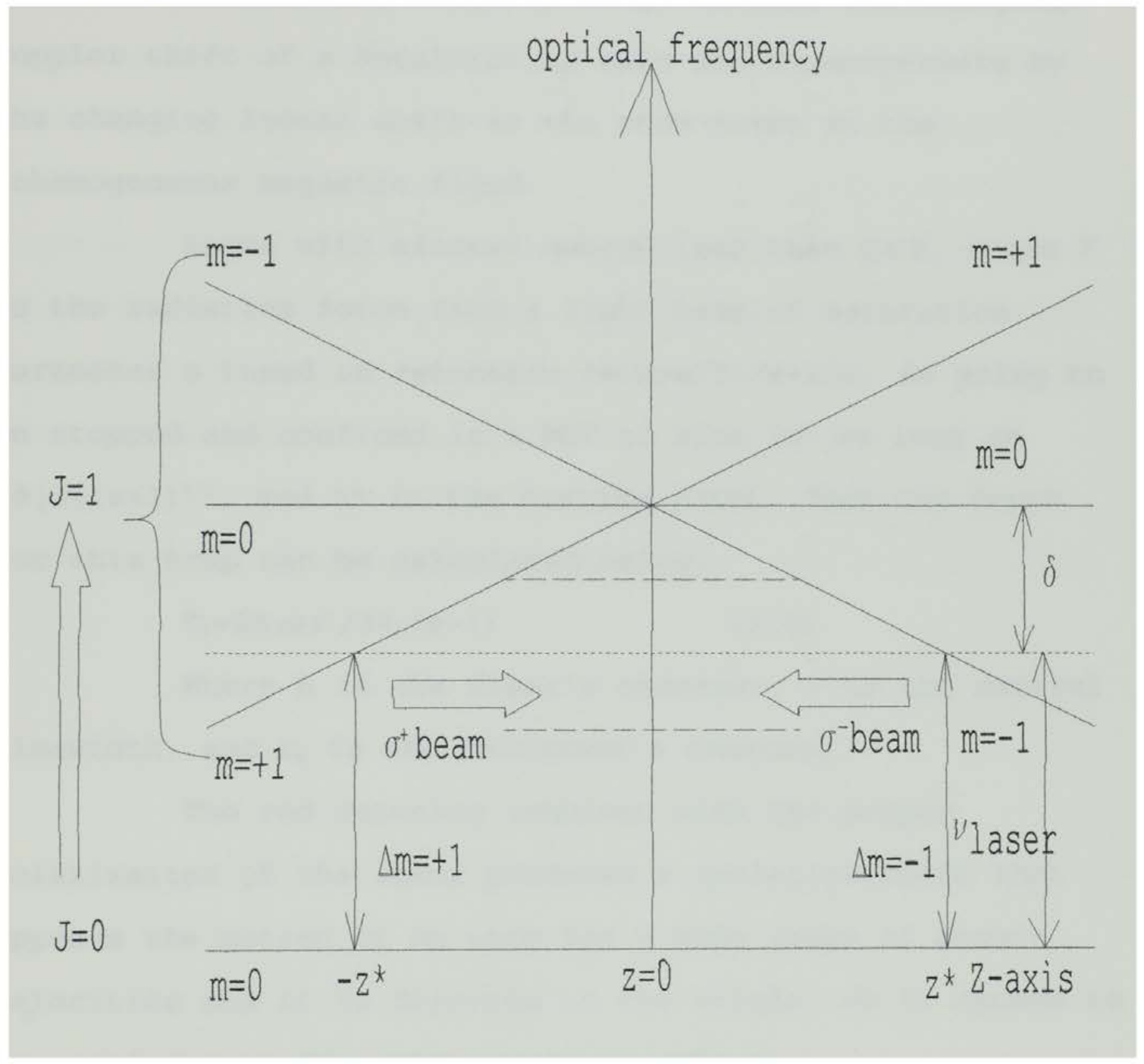

Fig 3.3. Arrangement for a MOT. The two dashed lines represent the frequency of opposing lasers seen by a moving atom. 
large detuning for the other, this fact together with the Doppler shift allows the resonance condition to occur inside the turning points. This makes the resonance possible for an extended distance and velocity range because the changing Doppler shift of a decelerating atom can be compensate by the changing Zeeman shift as the atom moves in the inhomogeneous magnetic field.

Atoms with kinetic energy less than $2 z^{*} F$, where $F$ is the radiative force from a light beam of saturation parameter $s$ tuned on resonance $(F=h \gamma s / 2 \lambda(s+1))$, is going to be stopped and confined in a МOT of size $2 z^{*}$ as long as $|\delta| \gg \gamma(s+1)^{1 / 2}$, and $z^{*}$ is the turning point. Then the depth for this trap can be calculated using:

$$
\mathrm{T}_{0}=2 \mathrm{~h} Y \mathrm{sz} * / 3 \mathrm{k}_{\mathrm{B}}(\mathrm{s}+1)
$$

Where $\mathrm{h}$ is the Plank's constant, $\mathrm{Y}$ is the natural linewidth, and $k_{\mathrm{B}}$ is the Boltzmann's constant.

The red detuning combined with the proper polarization of the laser produces a radiative force that opposes the motion of an atom for a wide range of atomic velocities and it is directed to the origin, so it oposes to any motion away from the origin as well. ${ }^{22}$

In the MOT atoms are cooled well below ordinary cryostat temperatures. It is possible to reach temperatures of the order of $T_{V}=\left(Y / Y_{c}\right) T_{D}$, where $Y_{c}=\hbar k^{2} / 2 M$ is the is the cooling rate for two level atoms in optical molasses, $M$ is the atoms mass.

In this experiment the trap is based on MOT the 
configuration described above. The only difference is in the coils that produce the desired magnetic field gradient. In our case a small solenoid was constructed to give the magnetic field profile required for the trap. The solenoid has four turns to the right (top part), a gap (about 1.25 times the radius of the solenoid) to create the region for the optical molasses and the four more turns to the left (bottom part), so that the same current flows in opposite direction for the top and the bottom parts of the solenoid. This construction gives a quadrupole field approximally the same as the one given by two coils. The magnetic field gradient is about 15 Gauss per centimeter, which is sufficient for trapping metastable atoms of Argon in a MOT. 


\section{Chapter IV- RADIATION SOURCE}

Lasers are particularly suitable for cooling atoms because due to their intrinsic characteristics. Laser light is highly monochromatic, unidirectional, intense and it is accessible in a wide range of optical frequencies. A cycling transition in the atom is necessary so that cycles of excitation and decay involve only two states. The excited atom will primarily decay back to the state from which the transition originated thereby leaving the atom ready for another absorption. The transition in Argon at $811.73 \mathrm{~nm}$ is in the near infrared and accessible with lasers.

The optical system required for cooling incorporates three main components. First is the light source. Second includes systems for evaluating the characteristics of the output light (i.e. power, linewidth) and spectrometers for monitoring the frequency. Finally, the optics that are used to direct the beam into the spectrometers and to introduce the laser beam into the molecular beam apparatus (MBA).

\section{A. LASER SYSTEMS}

The laser system is a three laser system, two titanium-sapphire ring lasers (Coherent 899-21) and an Argon Ion laser (Coherent Innova 200), which is used as a pumping laser for the ring lasers. The titanium- sapphire ring 
lasers used in these experiments are capable of a power output of up to two watts in ideal conditions. One of these lasers (RLI) is used in the cooling process of the metastable atoms emerging from the source and for the holeburning experiments. The hole-burning was also used as a tool that helped to check the alignment of the laser with the atomic beam, to measure the linewidth of the lasers, and its polarization. The other ring laser (RL2) is used to create the optical molasses for the MOT.

The ring laser system (Coherent $899-21$ ) is a solid state ring laser using a titanium-sapphire crystal as the gain medium. ${ }^{23}$ It is tunable from 680 to $1025 \mathrm{~nm}$. A high power pump laser, a $25 \mathrm{~W}$ Argon Ion laser (Coherent Innova 200), is used as the pump source. The system incorporates passive and active frequency control in order to obtain stable operation in a single longitudinal cavity mode.

All components and stages are mounted directly or indirectly to a 2 -inch diameter bar that provides mechanical strength and thermal stability. Unidirectional lasing is achieved with an optical diode. For passive frequency control a series of intracavity frequency filters are used. Active frequency control is achieved with an electronic servo loop and reference cavity. With $15 \mathrm{~W}$ in the pump beam, we obtain an output power of about $1.2 \mathrm{~W}$, and at the entrance of the atomic beam apparatus approximately $900 \mathrm{~mW}$. Using a high resolution Fabry-Perot interferometer the 
linewidth was measured to be about $20 \mathrm{MHz}$. Calculations of the laser linewidth from the hole-burning experiments, show $\Delta v \approx 18 \mathrm{MHz}$.

\section{B. ABSORPTION SPECTROMETERS}

Absorption experiments provide another simple way to determine the frequency characteristics of the laser. In the case of Argon, a metastable state $\left(1 S_{5} \mathrm{~J}=2\right)$ is the lower state of the cycling transition. The atoms in the metastable state are created in a gas discharge tube (GDT). Because of collisional processes, the atoms may move toward or away from the light source and the absorption line will be Doppler broadened.

To achieve the absorption and saturated absorption measurements, two "probe" laser beams A and B are directed through the discharge tube onto two photodiodes, as shown in figure 4.1 (respectively $D_{A}$ and $D_{B}$ ). Probe beam $A$ is used for the absorption measurement. The signal from photodiode $D_{A}$ is converted to a voltage by an I/V amplifier and displayed on a voltmeter. The voltmeter signal is highest when the light is not in resonance with an atomic transition (no absorption occurs) and lowest when the light frequency is in resonance with the frequency of the transition, so during the tuning process the minimum possible voltage output has to be obtained.

When the laser is tuned to a resonant frequency, 


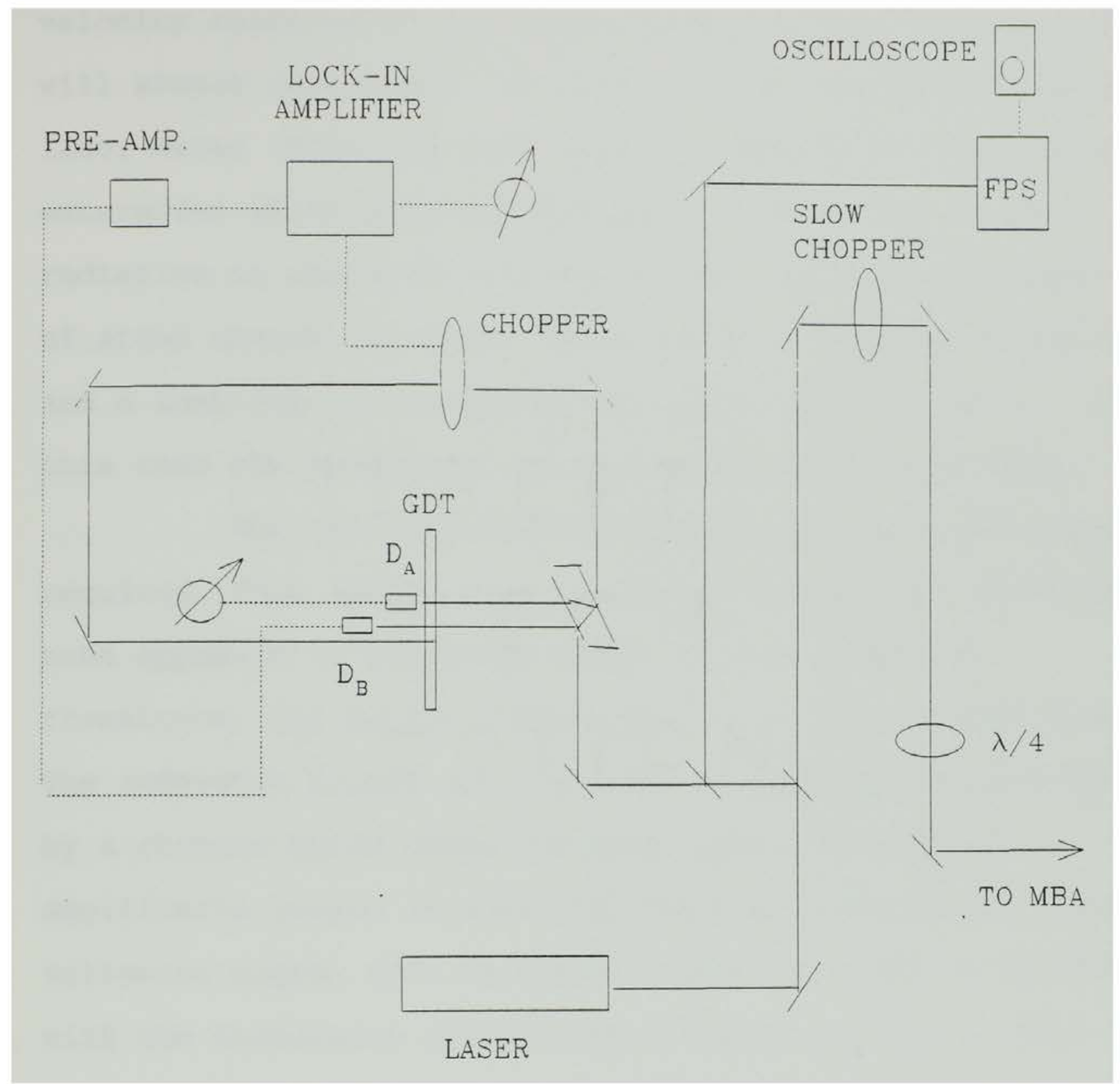

Fig. 4.1 Schematic of Optical Components of the Experiment. 
$v_{\text {rea }}$, the absorption will be maximum conly atoms with zero velocity relative to the light source in the discharge tube will absorb radiation). If there are two counterpropagating laser beams (from the same source), and one of them produces saturation after passing through the tube, then no more radiation is absorbed from the second one since the same set of atoms absorb the light. This causes a reduced absorption and a Lamb dip in the absorption curve centered on $v_{\text {res }}$; in this case the absorption of the second beam is minimum.

For the saturated absorption another laser beam is required. This "pump" beam (directed through the discharge tube opposite to the probe beam) will saturate the transition. The output from $D_{B}$ goes to a lock-in amplifier. The reference signal for the lock-in amplifier is provided by a chopper which chops the pump beam. The lock-in amplifier's output is also monitored by a voltmeter. This voltmeter signal will be maximum when the light is resonant with the transition and minimum when it is not. In this experiment the Lamb dip in the absorption curve is not observed due to the noise in the discharge tube and also because the linewidth of the laser is wider than the linewidth of the atomic transition.

When there is a maximum saturated absorption and a minimum absorption signals the laser is tuned to the resonant frequency of the cycling transition in Argon. 


\section{OPTICS FOR LASER BEAM DIRECTION}

The laser light from the pumping laser is split by a 50/50 plate beamsplitter and directed to the ring lasers, RL1 and RL2. The main beam from the RL1 is split with a microscope slide. The strong beam continues through a slow ( $1 \mathrm{~Hz}$ ) chopper and a 1/4-wave plate before being directed into the molecular beam apparatus (MBA). The slow chopper allows acquisition of laser on and laser of data at $.5 \mathrm{sec}$ intervals to eliminate effects due to instability of the atomic beam source. The 1/4-wave plate converts the linearly polarized light from the laser system into circularly polarized light $\left(\sigma^{+}\right)$.

The weak beam from the microscope slide is also split by another microscope slide. The weaker of these two, serves as input to a Fabry-Perot interferometer (FPS). The stronger is directed onto a thick beamsplitter. The reflections off the front and back facets of the beamsplitter are used as the probe beams in the absorption spectrometers. The beam that continues through the beamsplitter is reflected back into the gas discharge tube; this will be the pump beam for saturated absorption measurements. A diagram showing the spectrometers and the optics is shown in figure 4.1 .

The light from LR2 is split by a $30 / 70$ plate beamsplitter (BS), both beams pass through quaterwave plate that circular polarize them. Once polarized the reflected 
beam (70\% beam) is directed into a 50/50 cube beamsplitter (CBS), that splits it into two more beams; the transmited one is directed by mirrors to enter the trapping chamber from the top, to create the vertical beams of the molasses. The two beams from CBS are used to create the $x-y$ plane part of the molasses, directing both of them into the trapping chamber, one of them directly, and the other by using two mirrors. All the beams are positive circularly polarized $\left(\sigma^{+}\right)$before they enter the chamber, every beam's path ends in a mirror that reflects the laser light back into the chamber with negative circularly polarization $\left(\sigma^{-}\right)$. This whole process is shown in fig 4.2 .

All the mirrors used in directing the laser beam into the molecular beam apparatus and the trapping chamber are gold-coated to minimize power loss. Standard aluminum mirrors are used to reflect the pump beam in the saturated absorption spectrometer since power loss is not a major concern in this case. 


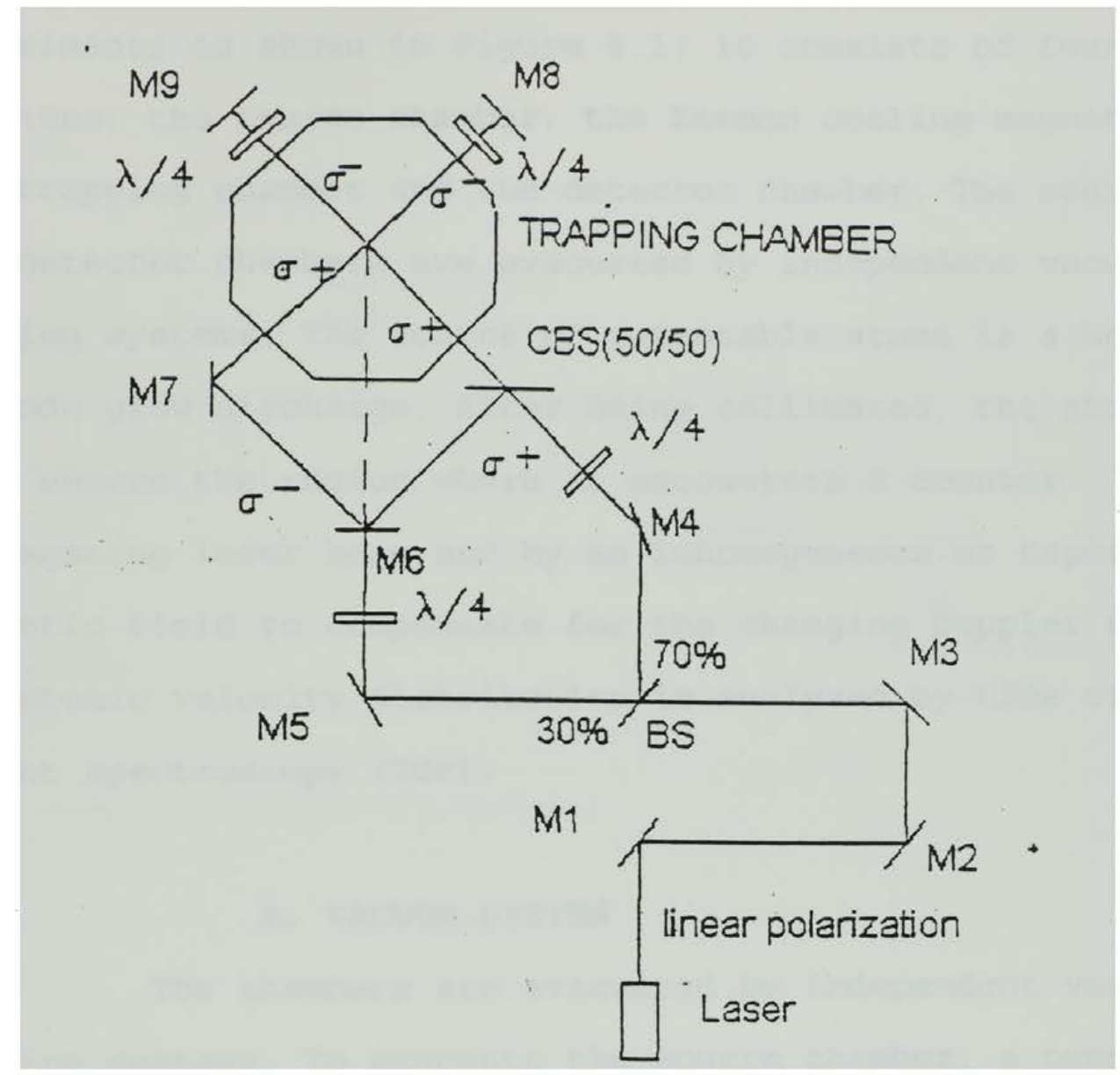

Fig. 4.2 Schematic of the optics to create the optical molasses 


\section{CHAPTER V - ATOMIC BEAM APPARATUS}

The atomic beam apparatus used in these experiments is shown in Figure 5.1; it consists of four main sections: the source chamber, the zeeman cooling magnets, the trapping chamber and the detector chamber. The source and detector chambers are evacuated by independent vacuum pumping systems. The source of metastable atoms is a hot cathode glow discharge. After being collimated, the atomic beam enters the region where it encounters a counter propagating laser beam and by an inhomogeneous or tapered magnetic field to compensate for the changing Doppler shift. The atomic velocity distribution is analyzed by time of flight spectroscopy (TOF).

\section{A. VACUUM SYSTEM}

The chambers are evacuated by independent vacuum pumping systems. To evacuate the source chamber, a pumping station consisting of a mechanical pump and a high vacuum oil diffusion pump is used. The mechanical pump is connected separately to the source chamber (roughing line), and to the foreline of the oil diffusion pump. It provides a roughing pressure of approximately 10 microns and, with the diffusion pump operating, provides a foreline pressure of 15 microns.

When the source is operating the pressure in the chamber is kept in the region of $5 \times 10^{-7}$ torr. With the 


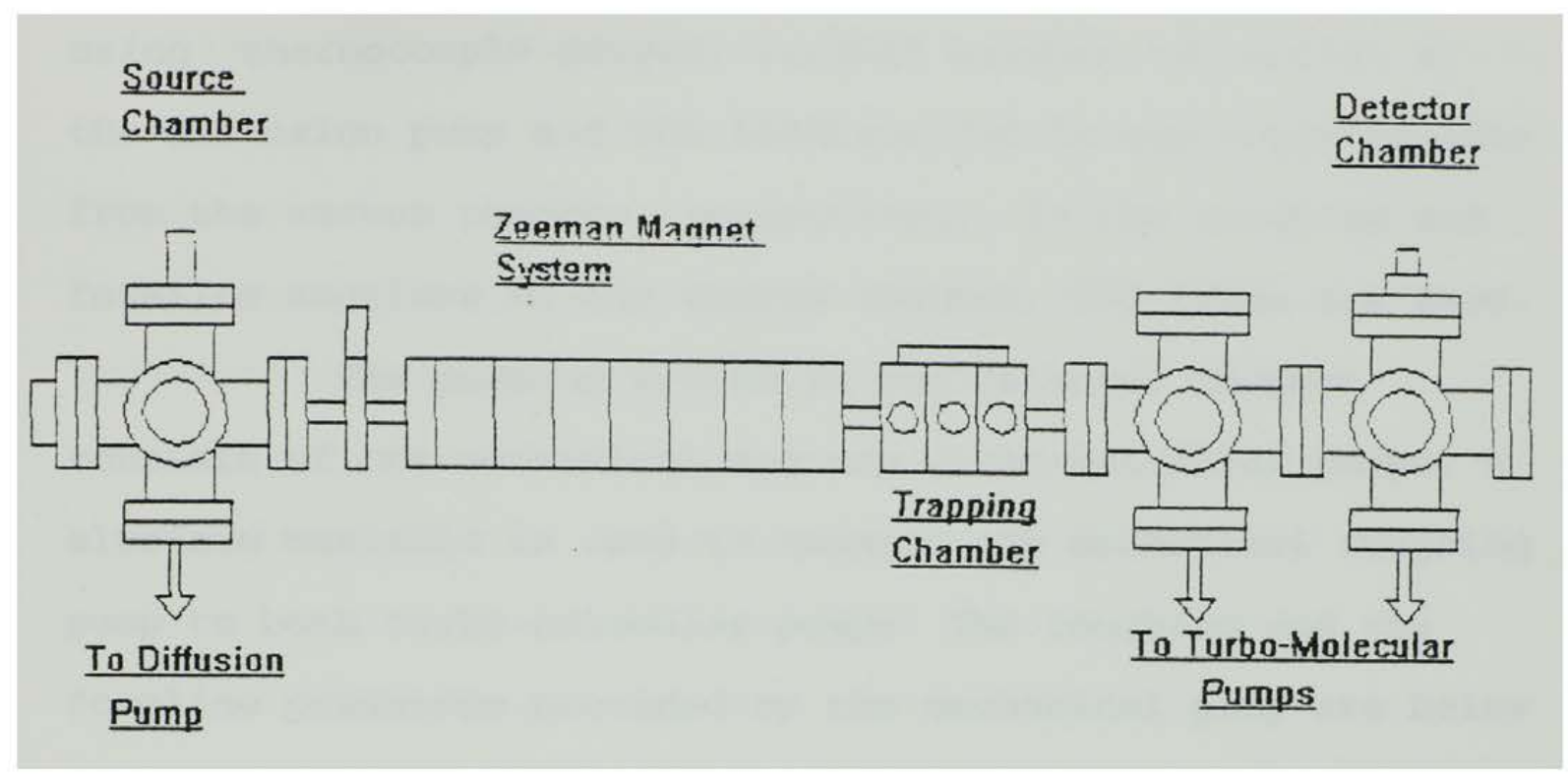

Fig 5.1. Molecular Eeam Apparatus (external view) 
source off, the pressure is about $8 \times 10^{-8}$ torr. A hot cathode ionization gauge is used to monitor the high vacuum pressure.

The foreline and roughing pressures are measured using thermocouple gauges, located between the outlet of the diffusion pump and the foreline and in the roughing line from the vacuum chamber, respectively. In the roughing and foreline sections of the source chamber, PVC lines are used. The pumping system in the detector chamber consists of one mechanical and two turbo-molecular pumps. A aluminum manifold is used to connect the mechanical roughing pump to both turbo-molecular pumps. The roughing and the foreline pressures provided by the mechanical pump are below 10 microns. This pressures are monitored by two thermocouple gauges, mounted on the top flange of the detector chamber and on the manifold, respectively. Hot filament ionization gauge is used to measure the high vacuum in this chamber. The working pressure in the chamber is maintained at about 7 $\mathrm{x} 10^{-7}$ torr. All high vacuum pumps are water-cooled.

\section{B. SOURCE and CHOPPER}

The source of the metastable atom beam is a Penning ionization discharge ${ }^{11}$. The $1000 \mathrm{~W}$, $500 \mathrm{~V}$ voltage power supply maintains about $50 \mathrm{~V}$ between the cathode and anode during typical operating conditions and supplies the approximately $400 \mathrm{~V}$ necessary to star the avalanching 
process necessary for starting the discharge. The cathode is a thoria-coated tungsten filament (with $2.5 \mathrm{~cm}$ length and $0.025 \mathrm{~mm}$ width) that produces electrons which are accelerated towards the anode by the potential difference across the discharge (between anode and cathode). The filament is coated by an electrolytic process with a solution of $340 \mathrm{cc}$ of $95 \%$ Ethylic alcohol, 0.4 grams of Thoria nitrate, and 14 gramsof Thoria oxideThe current used of $0.1 \mathrm{~A}$ is applied for approximately $45 \mathrm{sec}^{24}$. The collisions between electrons and argon gas form a plasma, a mixture of photons, metastable atoms, ions, neutral and Rydberg atoms, that effuse through a pinhole exit (.71 $\mathrm{mm}$ diameter) in the center of an aluminum anode at the end of $3 \mathrm{~cm}$ long Pyrex tube, where the discharge occurs. ${ }^{24}$ The filament current varies between $10-15$ $A$, and is used to control the discharge current, which typically is about $300 \mathrm{~mA}$.

The gas supplied to the discharge enters the tube through a servo-driven valve (Granville-Phillips series 216) which is part of an automatic pressure controller. The pressure in the discharge tube is monitored by a convectron gauge (Granville-Phillips series 275) and maintained at about 7 mTorr.

Intensity is especially important for a metastable state noble gas beam, since only a fraction of the total neutral beam is in the desired state. It has been shown, ${ }^{25}$ that a longitudinal magnetic field, applied to a low 
pressure, hot cathode glow discharge, will increase the intensity of metastable atoms effusing from a central anode slit. To provide this magnetic field, a solenoid with a magnet constant of 108 Gauss/A is mounted on the discharge tube such that the axial magnetic field constrains the discharge plasma along the axis between the filament and the exit slit. A high current on the source magnet will also increase the number of ions effusing from the source. This is an undesirable effect since the background will increase considerably when the magnet solenoid is turned on. For this reason, in place of the current that will maximize the yield of metastable atoms, a current of .6 A, corresponding to a maximum yield-background ratio is used on the source magnet. Once the discharge products effuse from the slit, they are continuously chopped by an aluminum wheel of 7.91 $\mathrm{cm}$ in diameter. The chopper has 2 diametrically opposed $0.081 \mathrm{~cm}$ slits and is driven by a synchronous motor at a frequency of $60 \mathrm{~Hz}$. The chopper slit and rotational speed give an open time about $80 \mu \mathrm{sec}$ and a beam pulse rate of 120 $\mathrm{Hz}$, which allows sufficient time $(8.3 \mathrm{msec})$ to observe nearly the complete thermal time-of-flight (TOF) metastable atom distribution in each pulse. After passing through the chopper, the beam is collimated with a $.009 \mathrm{~cm}$ slit.

When the chopper is open, the light from a lightemitting diode (LED) stimulates a photo-transistor. The LED and the photo-transistor are located at opposite sides of 
the chopper wheel, such that, the photo-transistor is stimulated only when the chopper is open. The signal generated by the transistor is amplified and delayed in order to assure coincidence with photoelectron pulses from the ultraviolet (UV) photons which come down the beam path when the chopper opens. This signal is used as a reference or start signal for the TOF spectroscopy. A pair of sweep plates, operating at $300 \mathrm{~V} / \mathrm{cm}$ normal to the beam, remove charged particles and ionize atoms in long-lived Rydberg states, leaving only metastable and neutral atoms in the ground state within the beam.

The trapping chamber has an octagonal shape cross section with $18 \mathrm{~cm}$ length and width and is $10 \mathrm{~cm}$ high. Port windows are mounted on six of the eight sides of the chamber, two more are on the top and bottom flange, respectively. The top flange is used as a holder for the magnet coil that provides the necessary magnetic field gradient for the MOT. The current in the coil is provided by a 60 A power supply.

\section{DETECTOR AND TIME OF FLIGHT ANALYSIS}

At the end of the flight path, photons and atoms are detected by measuring electrons ejected from a gold mirror by either the photoelectric effect or Auger effect. These electrons are collected and amplified by a channeltron electron multiplier. The detector and the gold mirror are 
mounted on an aluminum plate, that is supported by two fixed rods and is connected to a linear motion feedthrough. The gold mirror is negative biased relative to the entrance cone of the electron multiplier; consequently, the ejected electrons are attracted into the electron multiplier. This entrance cone is biased at -2000 volts and the exit of the detector is operated at -200 volts. The gold mirror is used as a source of electrons as well as a reflecting mirror for the laser. The laser enters the detector chamber through a vacuum port window and is directed antiparallel to the atom beam after reflecting from the gold mirror. As a result, the beam is irradiated by the laser along the entire flight path $(1.824 \mathrm{~m})$ from the source exit slit to the gold mirror in the detector chamber.

The beam path length guarantees sufficient TOF for all excited atoms to undergo optical decay before reaching the detector (except for the metastable atoms). On the other hand, only metastable atoms and photons have sufficient energy to eject electrons from a gold mirror. The groundstate atoms in the beam (unless they are product of the dissociative recombination in the source) have kinetic energy less than the work function of the gold (5.1 ev), and therefore are not detected.

\section{DATA ACQUISITION}

The signal from the electron multiplier is pulse- 
shaped and amplified by a pre-amplifier (Canberra model 2005) and then by a spectroscopy amplifier (Canberra model 1413). A constant timing single channel analyzer (Canberra model2035A) reduces the background noise, and gives the input pulses to the ORTEC ACE Multichannel scaler (a hardware and software package that performs data collection and MCS emulation) operating in a Zenith personal computer. The detection system measures the arrival time of the metastable atoms and photons. The TOF spectra of the metastable atoms are recorded in channels $40 \mu \mathrm{sec}$ wide and the sweep length is 200 channels (the time/channel is 40 $\mu \mathrm{sec})$. Since the path length is known, the velocity distribution of the metastable atoms can be obtained as a function of their TOF. The velocity resolution of the TOF method is only limited by the chopper open time. For $\Delta t_{c}=80$ usec, $\Delta \mathrm{v}=\mathrm{v} /(1+.4 \mathrm{x}$ Channel $)$. For velocity $400 \mathrm{~m} / \mathrm{s}$, the resolution is $\Delta v=8.6 \mathrm{~m} / \mathrm{s}$. As the atom's velocity decreases, the resolution of the method is better. A typical TOF distribution is shown in figure 5.2. Metastable atoms in the beam that effuse from a Maxwellian velocity distribution in the discharge have a TOF distribution $f(t)$ normalized to $\mathrm{N}$ total counts is given by: ${ }^{27}$

$$
f(t)=\frac{C}{t^{5}} \exp \left[-\frac{5}{2}\left(\frac{t_{p}}{t}\right)^{2}\right] \quad(5.1)
$$

Where $t_{p}=\mathrm{mL}^{2} / 5 \mathrm{kT}$ is the most probable TOF, $\mathrm{L}$ is 


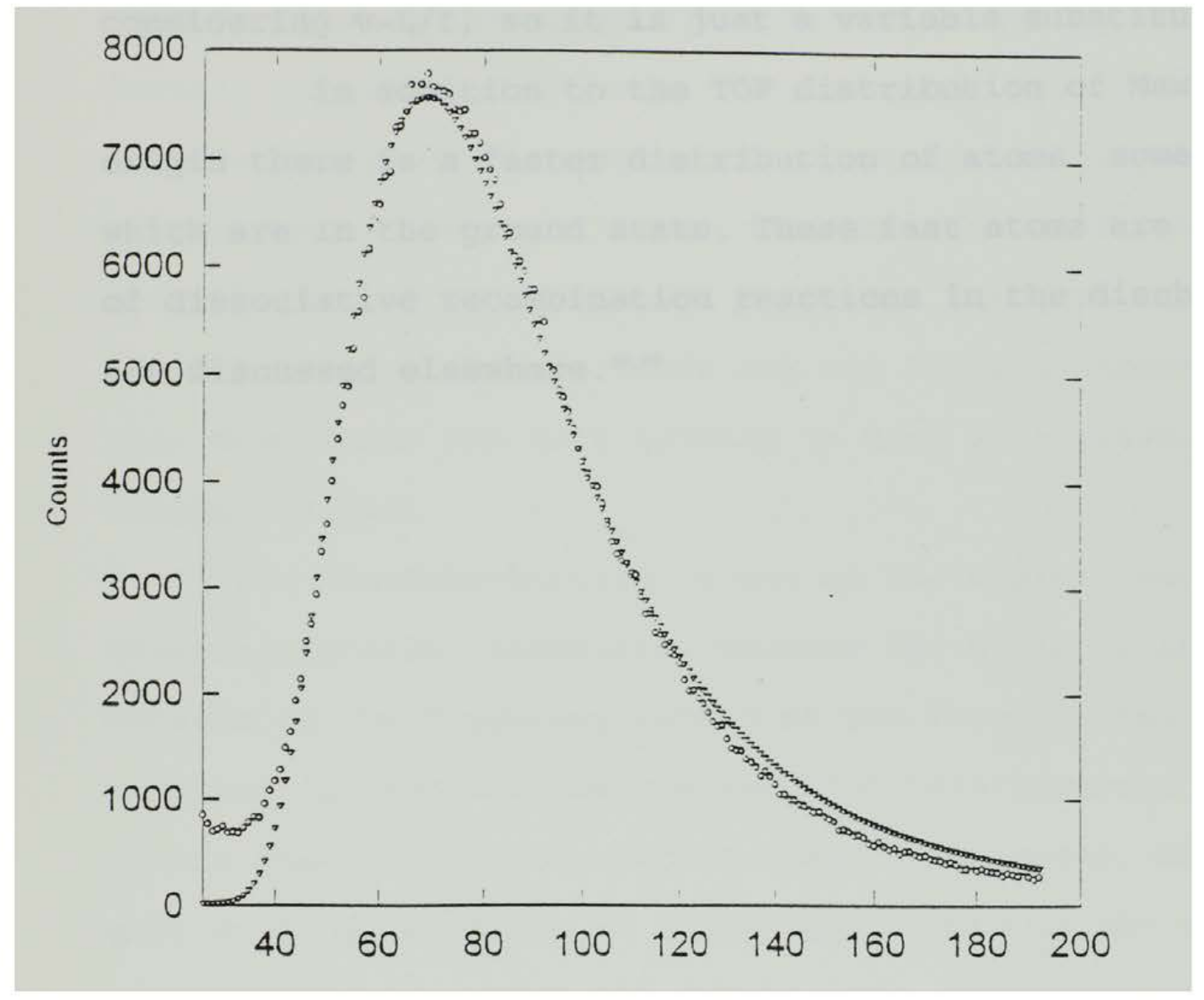

Figure 5.2 Typical TOF Distribution of metastable Argon atoms (Hollow circles). The time/channel is $40 \mathrm{msec}$. triangles show the distribution fitted to eq. 5.1.. 
the flight path and $C$ is the normalization constant, $\mathrm{C}=(25 \mathrm{~N} / 2) t_{\mathrm{p}}{ }^{4}$.

The TOF distribution can be easily obtained from Maxwellian velocity distribution for an effusion process by considering $v=L / t$, so it is just a variable substitution.

In addition to the TOF distribution of Maxwellian origin there is a faster distribution of atoms some of which are in the ground state. These fast atoms are products of dissociative recombination reactions in the discharge and are discussed elsewhere. ${ }^{28,29}$ 


\section{CHAPTER VI. ATOMIC COOLING EXPERIMENTS}

\section{A. HOLE-BURNING EXPERIMENTS}

The first clear experimental demonstration of laser cooling of an atomic beam was hole-burning. Hole burning is achieved by either detuning the laser frequency to a frequency corresponding to a certain velocity on the velocity distribution (in our case the time of flight distribution of metastables), or zeeman shifting the atomic transitions, so that so atoms see the laser in resonance. In this experiment the hole-burning is used as a measuring and diagnostic tool.

The hole-burning is one of the easiest ways to measure the laser linewidth, because the width of the hole depends on the frequency spread of the laser. Laser polarization can also be checked with hole-burning, in fact, it was the only method used in these experiments. One of the most critical problems of these experiments is the alignment of the laser beam with the atomic beam. Alignment problems are successfully detected by hole-burning since the depth of the hole is direct result of the alignment, the deeper the hole in the TOF distribution the better the alignment is between the laser and the atomic beam.

Atoms moving toward the light source tuned to resonance will see the light Doppler shifted to the blue $(\delta>0)$, to compensate for this the laser should be detuned to 
the red side of the transition frequency $(\delta<0)$. Atoms with velocity $\mathrm{V}$ will be in resonance with the laser, thus, burning a hole in the TOF at a channel corresponding to the velocity $\mathrm{V}(\operatorname{chx} 40 \mu \mathrm{sec}=1.824 / \mathrm{V})$. If the detuning $\delta$ is compensating for the Doppler shift corresponding to a velocity $\mathrm{v}\left(\delta=v_{\text {res }} \mathrm{V} / \mathrm{c}\right)$, atoms with velocity $\mathrm{V}$ will undergo several collisions and decelerate until they are taken out of resonance, this will appear as a dip in the velocity distribution at the initial velocity of these atoms and a corresponding bump at velocity for which they went out of resonance.

Before every cooling experiment, hole-burning measurements were taken in order to calibrate the laser frequency and to check the alignment of the laser with the atomic beam. The intensity of laser light is always greater, than the saturation intensity of the cycling transition for Argon $\left(I_{\text {ваt }}=8.2 \mathrm{~mW} / \mathrm{cm}^{2}\right)$. Different detunings were used in hole-burning. The dips in the TOF distribution were observed at the velocities corresponding to the laser detuning, and also observed were the bumps at later times corresponding to the lower velocities in the distribution.

To determine the laser linewidth the hole-burning data is fitted, using the least square method. Subtracting the hole-burning spectra from the fitted absorption curve a difference spectrum is obtained and measuring the FWHM (full wave half maximum) of the difference spectrum, the 
frequency spread of the laser can be determined. All these result are shown in table VI.1 and in fig 6.1-4(a) and (b).

\begin{tabular}{||c|c|c|c|c|c||}
\hline $\mathrm{Ch}$ & $\delta(\mathrm{MHz})$ & $\mathrm{v}(\mathrm{m} / \mathrm{sec})$ & $\mathrm{LW}(\mathrm{MHZ})$ & TOF (ms) & Figure \\
\hline 88 & 639 & 518 & 36 & 3.52 & 6.1 \\
\hline 93 & 605 & 490 & 45 & 3.72 & 6.2 \\
\hline 96 & 586 & 475 & 51 & 3.84 & 6.3 \\
\hline 96 & 586 & 475 & 34 & 3.84 & 6.4 \\
\hline
\end{tabular}

Table VI.1. Ch is the channel corresponding to a velocity $\mathrm{V}$ at which the hole-burning should be centered; $\delta$ is the laser detuning; $L W$ is the linewidth of the laser; and time is given by $\mathrm{Chx} 40 \mu \mathrm{sec}$. Comparing the results from figures $6.3 \mathrm{~b}$ and $6.4 \mathrm{~b}$, the power-broadened effect can be shown since in these two spectra the linewidth was measured for different laser power, $1.2 \mathrm{~W}$ and .9W respectively, and linewidth is less with less power output.

The Zeeman effect can be used to compensate for the Doppler shift so it is also possible to do hole-burning by keeping the laser tuned to the resonance and shifting the atomic transition frequency with a uniform magnetic field. In this case the position of the hole depends on the magnitude field and the polarization of the laser. Table VI.2 shows different velocities, at which the hole appears in the TOF distribution, corresponding to different magnitudes of the magnetic field. 


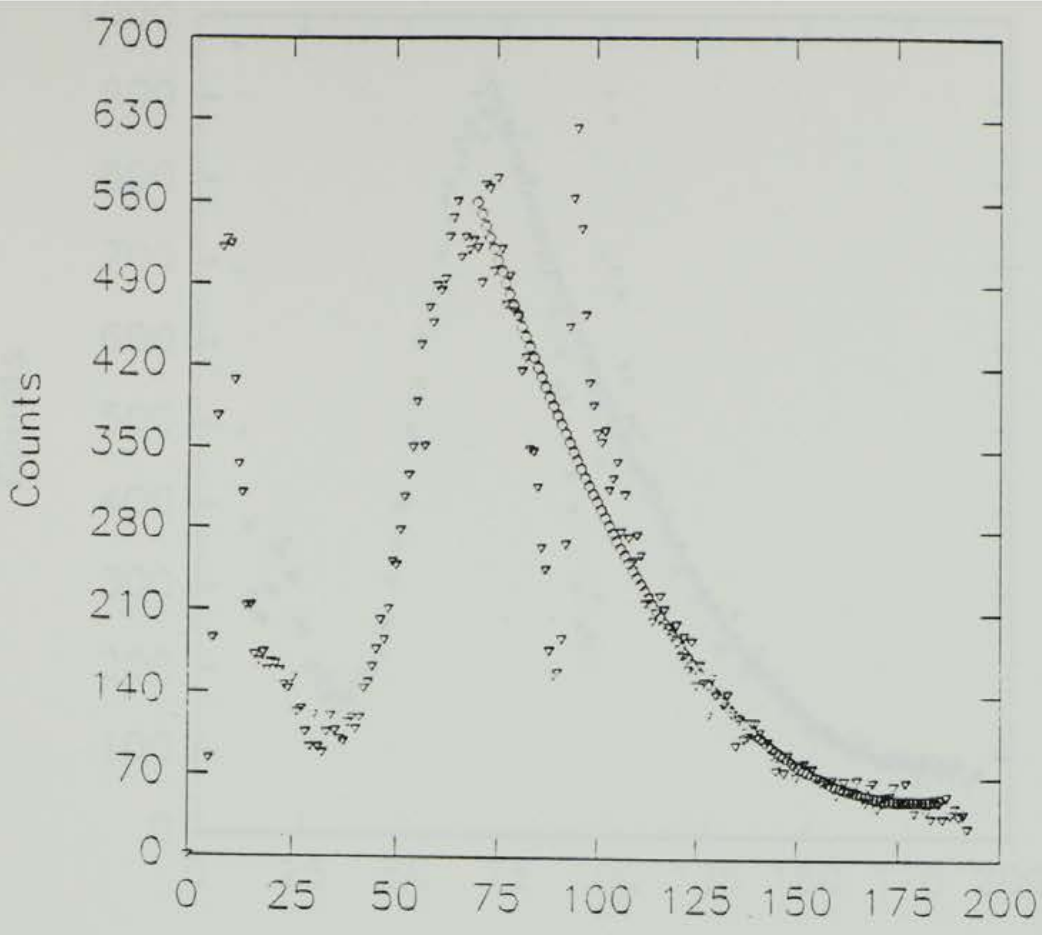

Channels

fig 6.1a.Circles show the fitted data, triangles show the hole-burning spectrum.

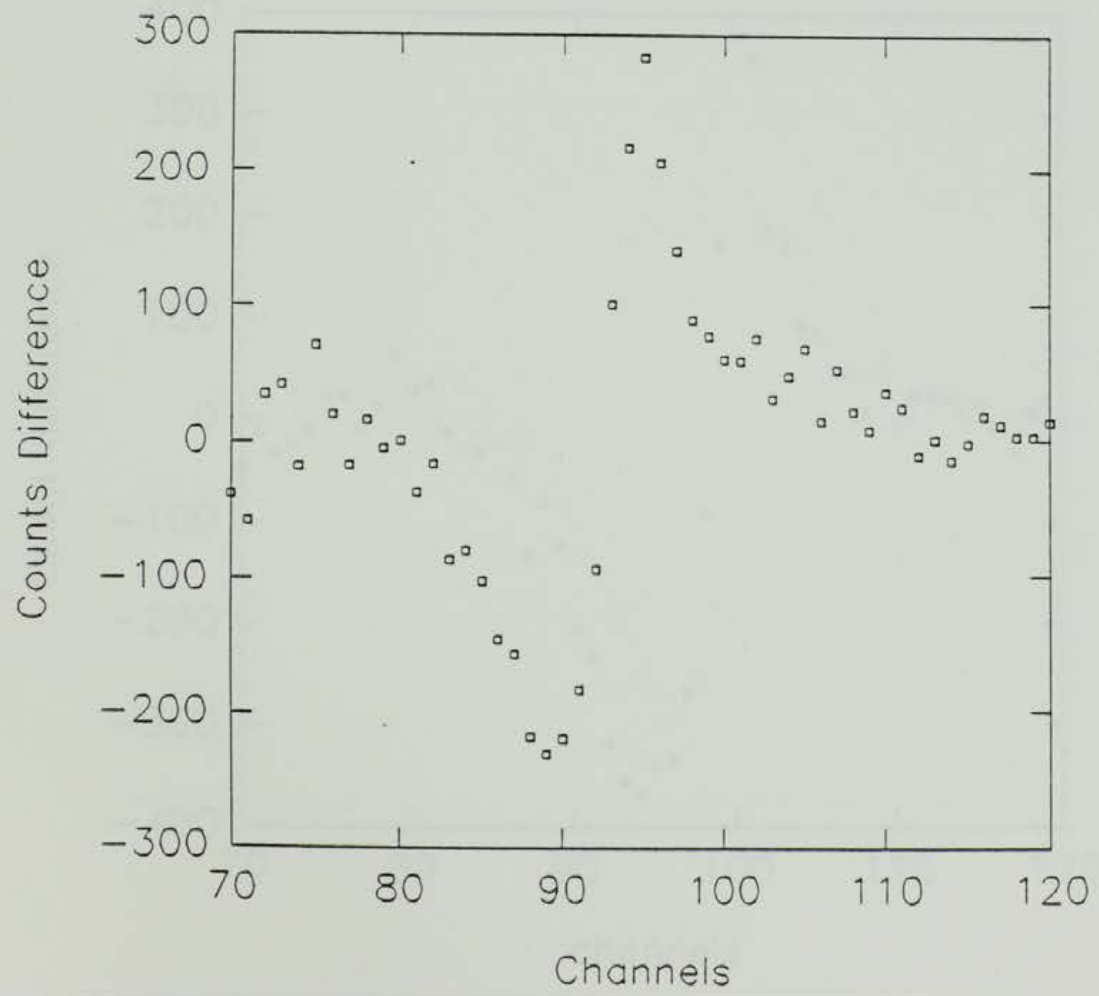

fig $6.1 \mathrm{~b}$. The absorption curve 


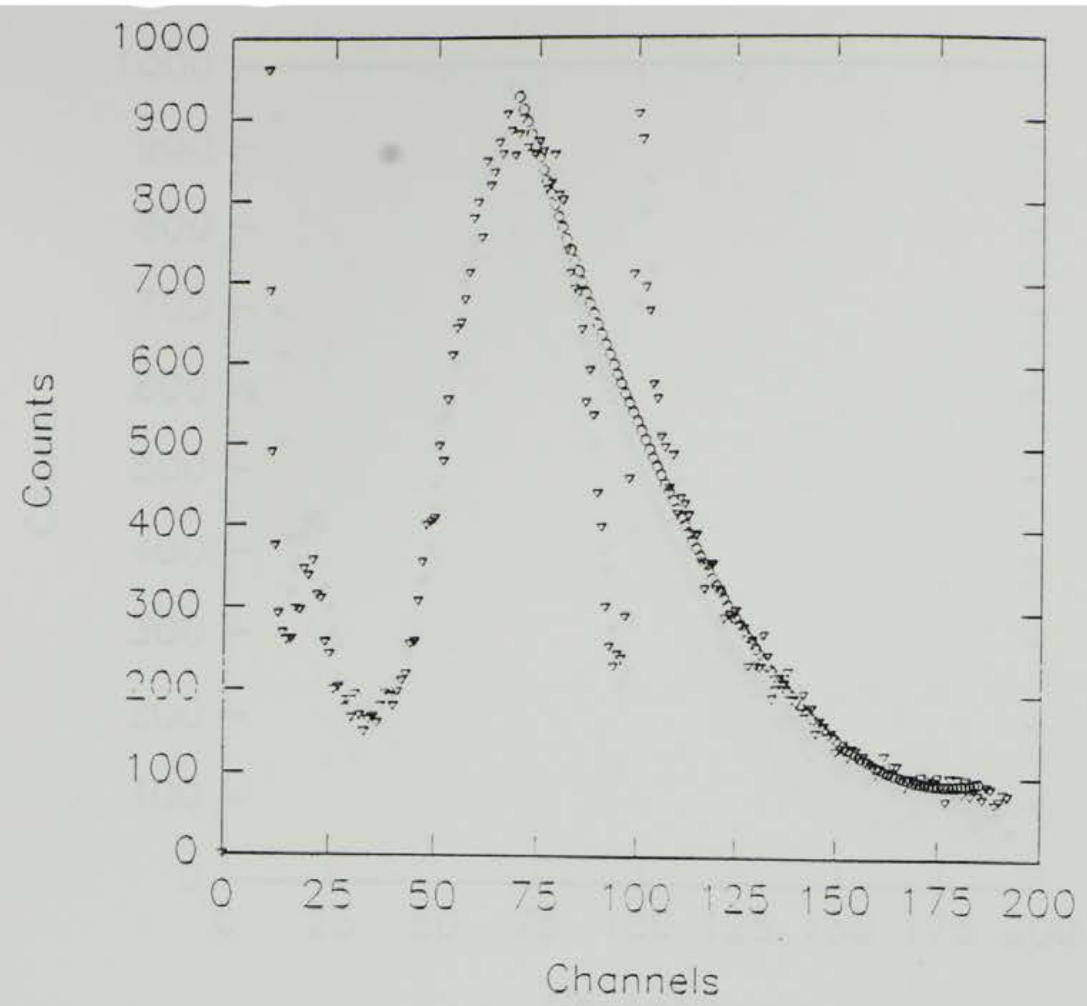

fig 6.2a. Circles show the fitted data, triangles show the hole-burning spectrum

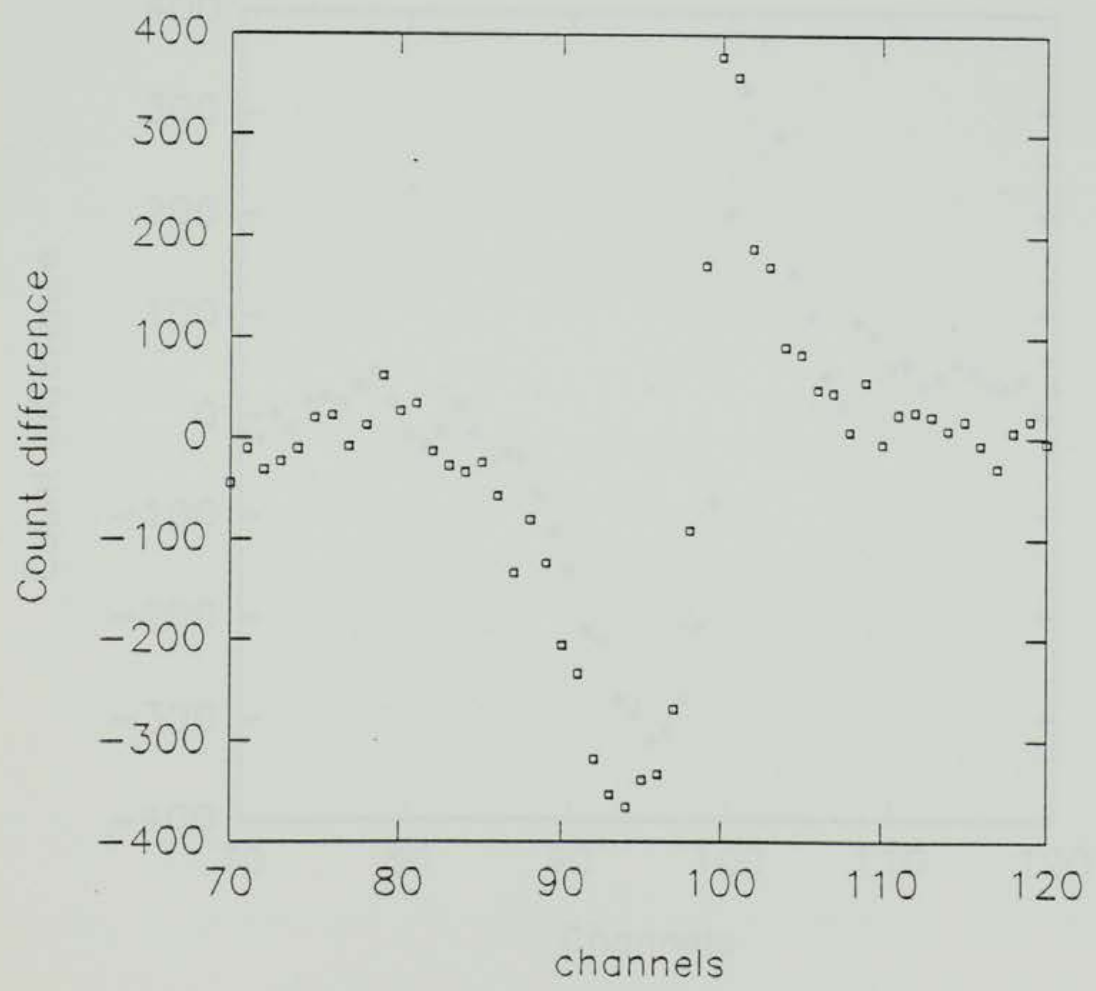

fig 6.2b. The absorption curve 


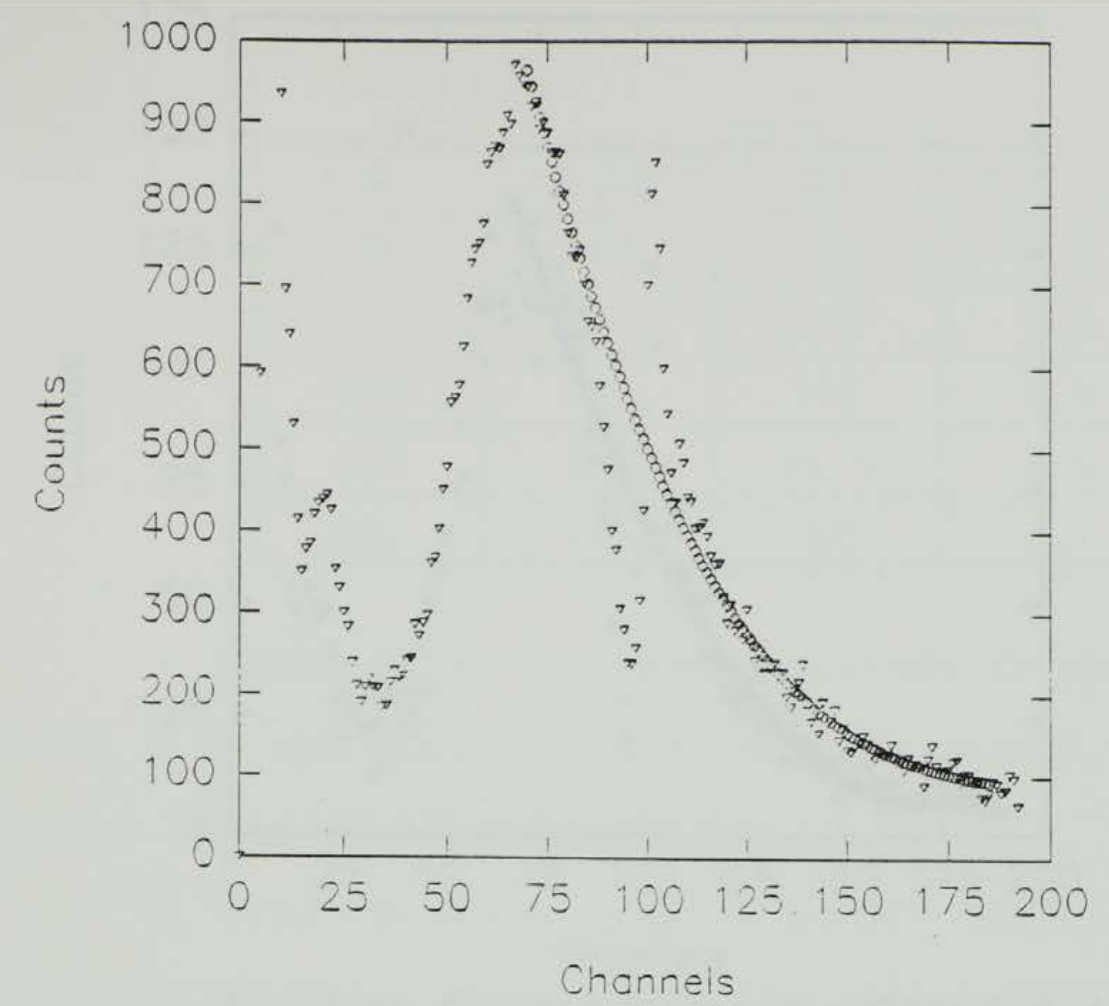

fig $6.3 a$ circles show the fitted data, triangle show the hole-burning spectrum

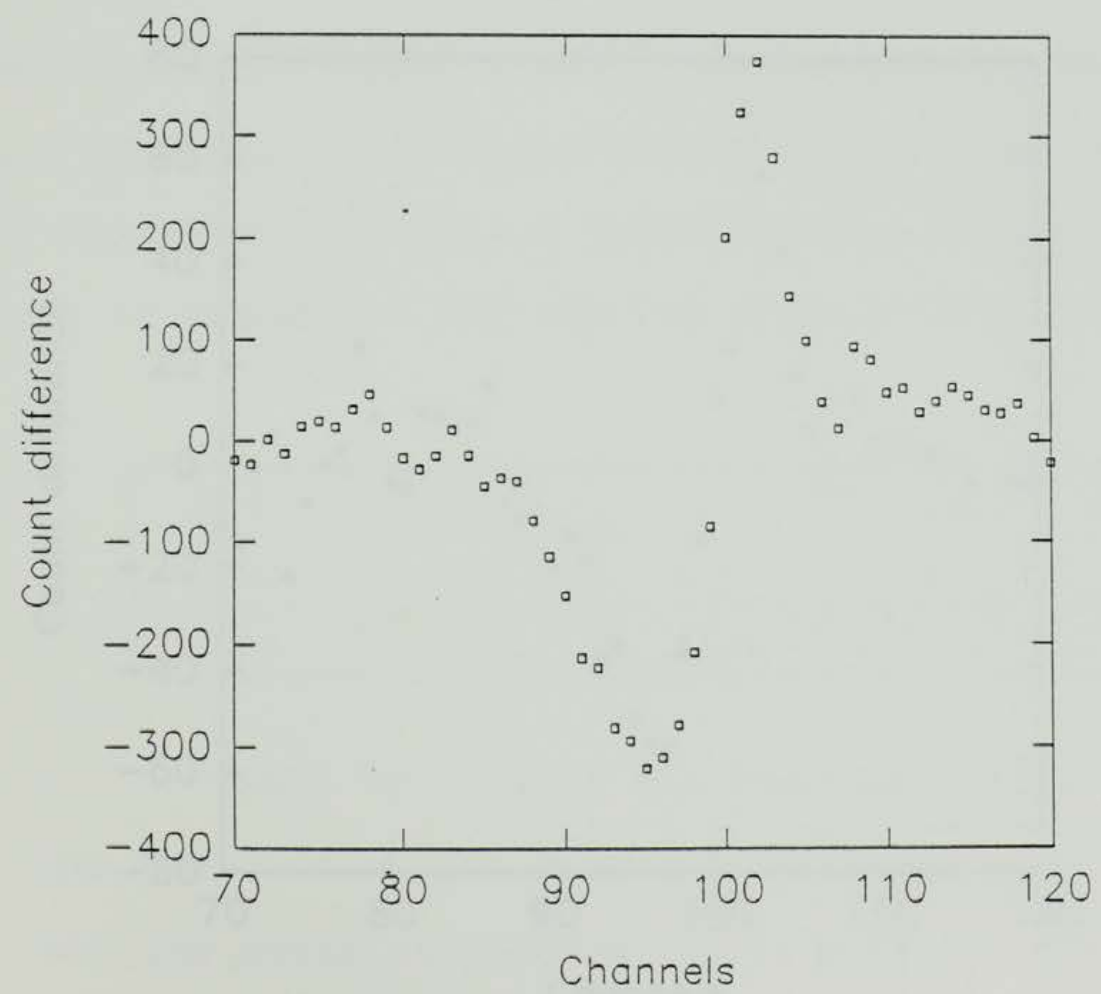

fig6.3b The absorption curve 


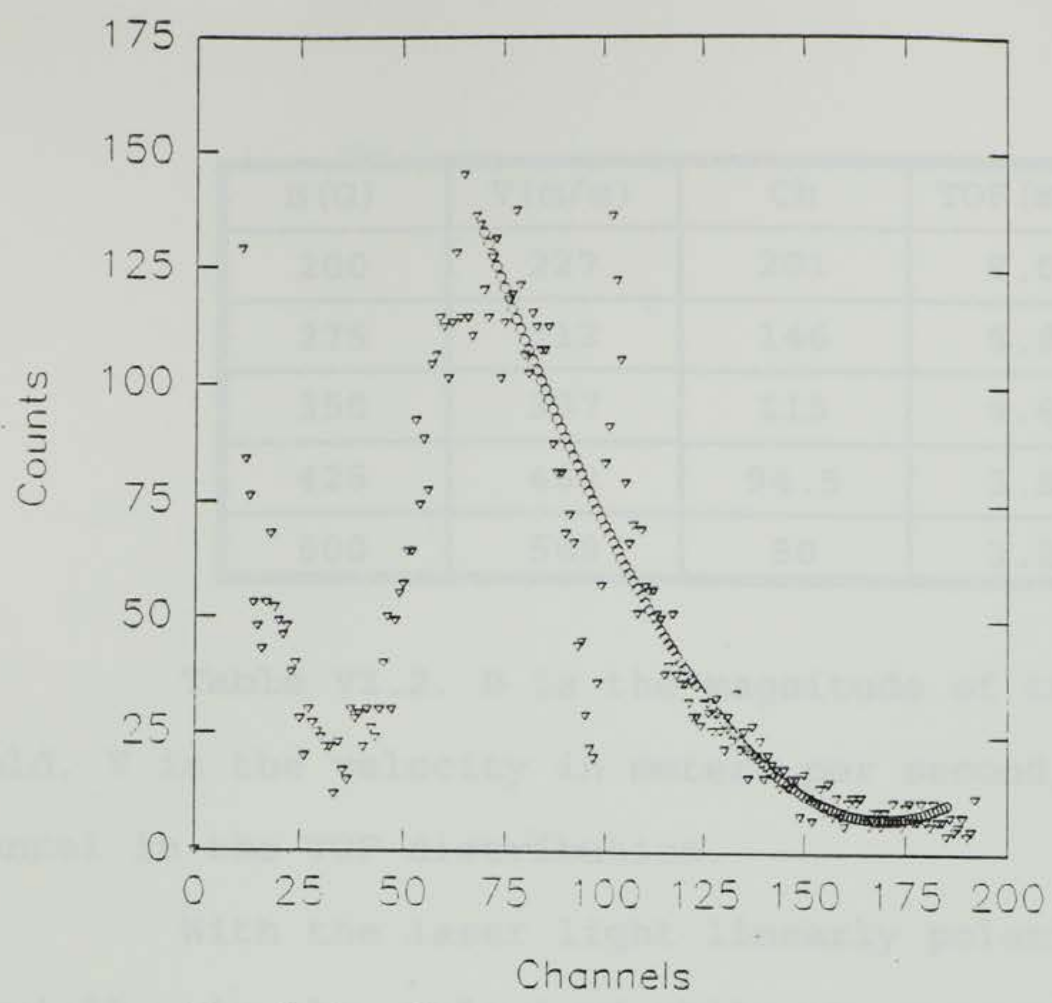

fig 6.4a Circles show the fitted data, triangles show the hole burning spectrum.

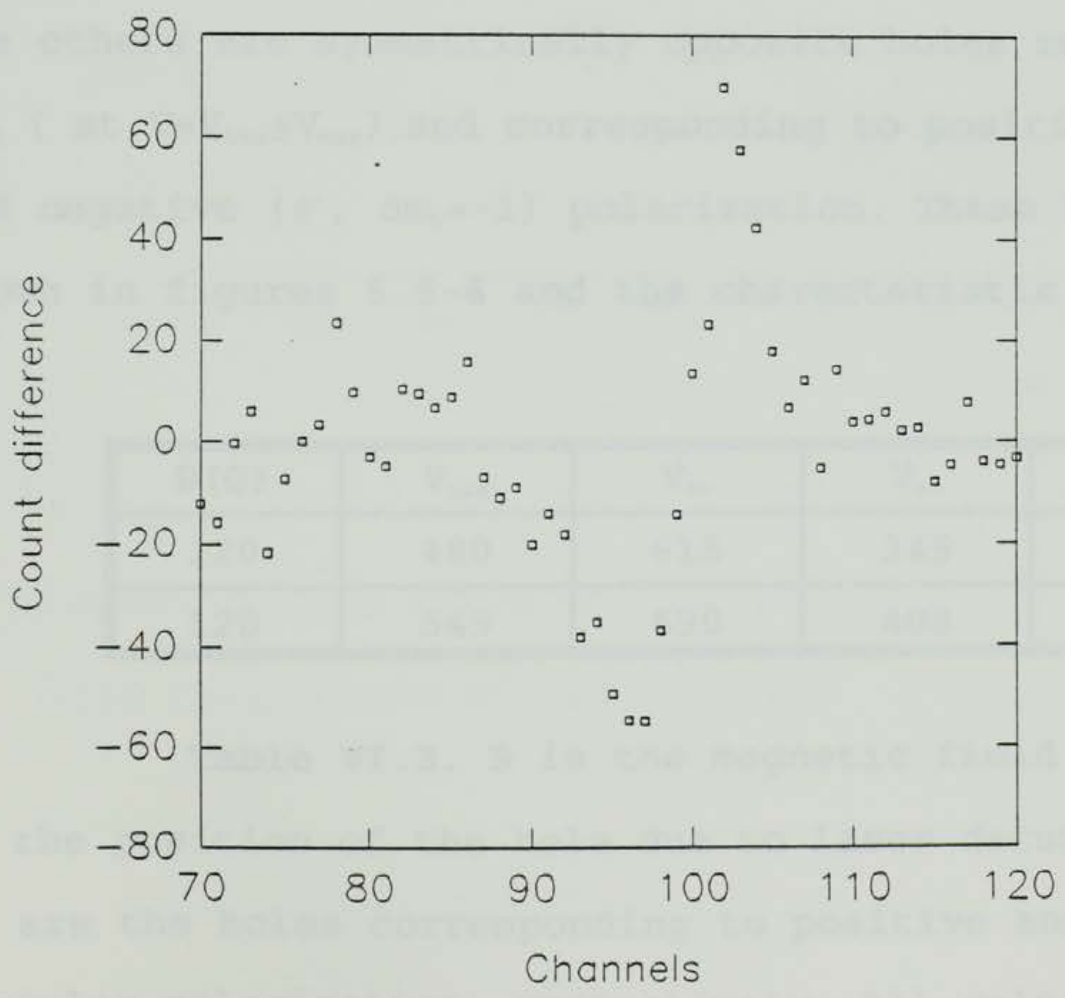

fig $6.4 \mathrm{~b}$ The absorption curve 


\begin{tabular}{||c|c|c|c||}
\hline $\mathrm{B}(\mathrm{G})$ & $\mathrm{V}(\mathrm{m} / \mathrm{s})$ & $\mathrm{Ch}$ & TOF $(\mathrm{ms})$ \\
\hline 200 & 227 & 201 & 8.0 \\
\hline 275 & 312 & 146 & 5.8 \\
\hline 350 & 397 & 115 & 4.6 \\
\hline 425 & 482 & 94.5 & 3.8 \\
\hline 500 & 568 & 80 & 3.2 \\
\hline
\end{tabular}

Table VI.2. B is the magnitude of the magnetic field, $V$ is the velocity in meters per second, Ch the channel in the TOF distribution.

With the laser light linearly polarized or partially circular polarized while the magnetic field is constant, three different holes will be burned in the distribution, one of them corresponding to the laser at $V_{\text {lab }}$ the others are symmetrically opposite holes relative to the $\mathrm{V}_{\text {las }}$ ( at $\mathrm{V}=\mathrm{V}_{\text {las }} \pm \mathrm{V}_{\text {mag }}$ ) and corresponding to positive $\left(\sigma^{+}, \Delta \mathrm{m}_{\mathrm{j}}=+1\right)$ and negative $\left(\sigma^{-}, \Delta m_{j}=-1\right)$ polarization. These results are shown in figures $6.5-6$ and the characteristic in table VI.3.

\begin{tabular}{|c|c|c|c|c||}
\hline $\mathrm{B}(\mathrm{G})$ & $\mathrm{V}_{1 \mathrm{as}}$ & $\mathrm{V}_{\sigma+}$ & $\mathrm{V}_{\sigma .}$ & figure \\
\hline 120 & 480 & 615 & 345 & 6.5 \\
\hline 120 & 549 & 690 & 408 & 6.6 \\
\hline
\end{tabular}

Table VI.3. B is the magnetic field in Gauss, $V_{\text {las }}$ is the position of the hole due to laser detuning, $V_{\sigma_{+}}$and $\mathrm{V}_{\sigma}$. are the holes corresponding to positive and negative circular polarization, respectively. All velocities are measured in meters per second. 


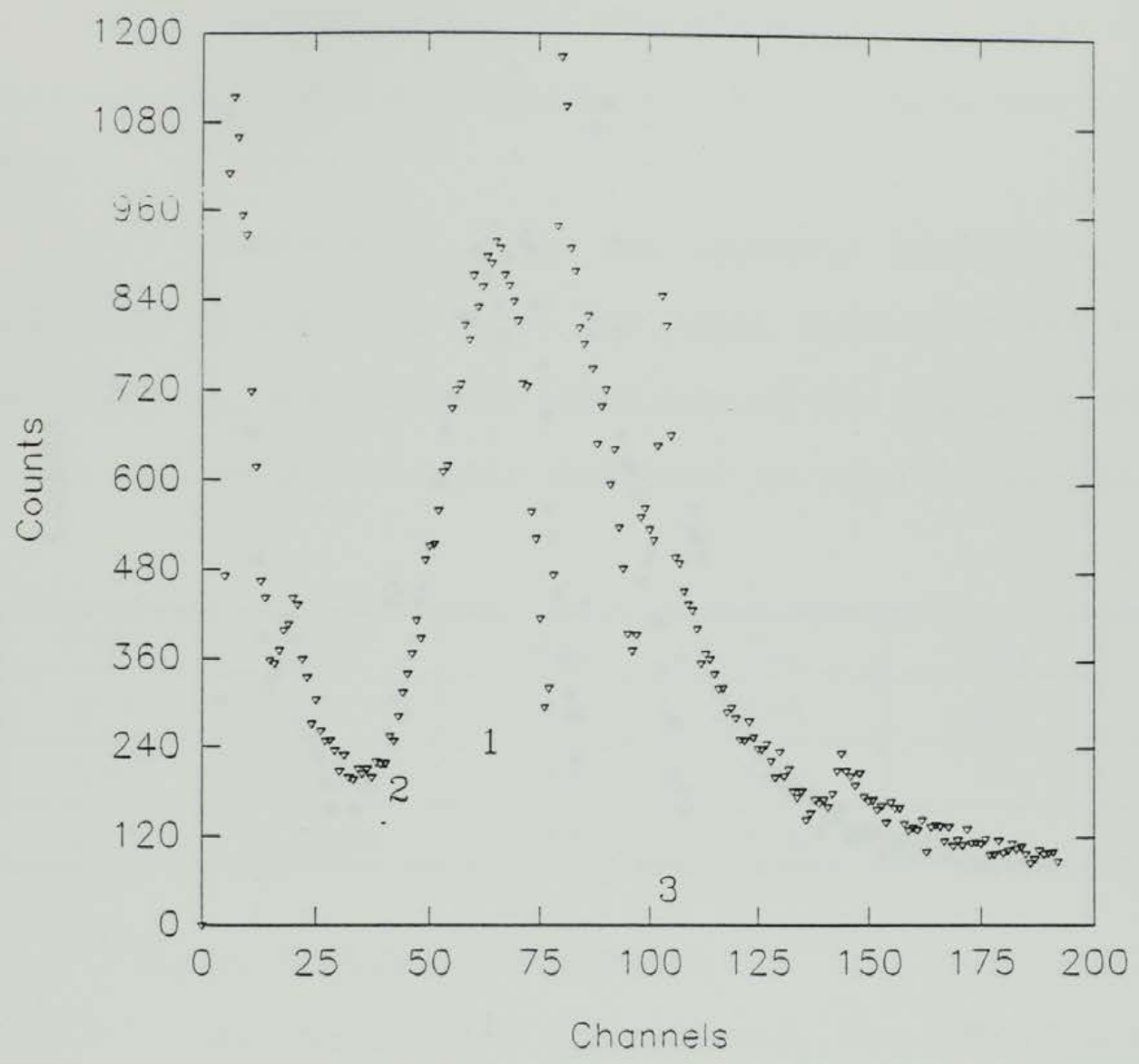

fig 6.5. Hole by the laser_1, laser detuning is $592 \mathrm{Mhz}$ magnetic field holes: $\sigma^{+}{ }_{2}^{2}$ and $\sigma^{-}{ }_{3} 3$, magnetic field magnitude of 120 Gauuss. 


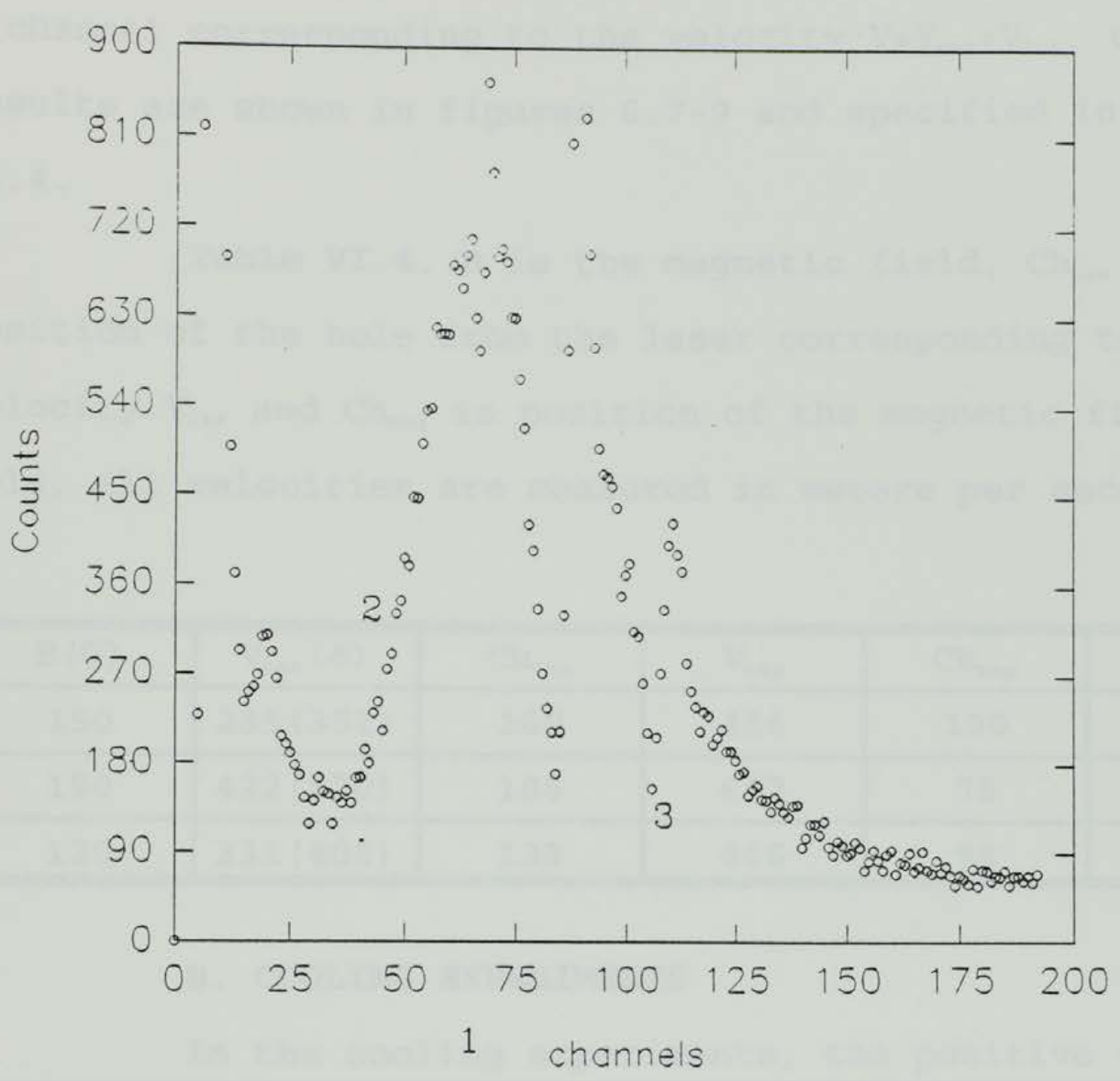

fig 6.6. Hole by the laser_1, the laser detuning is $678 \mathrm{Mhz}$ magnetic field holes: $\sigma^{+} \_2$ and $\sigma^{-} \_3$, magnetic field magnitude of 120 Gauss. 
In case of positive circularly polarized light (the polarization used for laser cooling) two holes are burned into the TOF distribution, one corresponding to the laser and the other due to magnetic field, that should be in a channel corresponding to the velocity $\mathrm{V}=\mathrm{V}_{\text {mag }}+\mathrm{V}_{1 \mathrm{as}}$, these results are shown in figures $6.7-9$ and specified in table VI. 4 .

Table VI.4. B is the magnetic field, $\mathrm{Ch}_{1 \mathrm{as}}$ is the position of the hole from the laser corresponding to the velocity $\mathrm{V}_{1 \mathrm{as}}$ and $\mathrm{Ch}_{\operatorname{mag}}$ is position of the magnetic field hole, all velocities are measured in meters per second.

\begin{tabular}{||c|c|c|c|c|c||}
\hline \hline $\mathrm{B}(\mathrm{G})$ & $\mathrm{V}_{1 \mathrm{as}}(\delta)$ & $\mathrm{Ch}_{\mathrm{las}}$ & $\mathrm{V}_{\operatorname{mag}}$ & $\mathrm{Ch}_{\operatorname{mag}}$ & figure \\
\hline 150 & $285(351)$ & 160 & 456 & 100 & 6.7 \\
\hline 190 & $422(520)$ & 108 & 642 & 78 & 6.8 \\
\hline 120 & $331(408)$ & 138 & 466 & 98 & 6.9 \\
\hline
\end{tabular}

\section{B. COOLING EXPERIMENTS}

In the cooling experiments, the positive circular polarized laser light is tuned to the resonance frequency, and the magnetic field is tapered, so that, the atoms with velocities equal to or less than the initial velocity $\left(V_{0}\right)$ are stopped, where $V_{0}$ is determined by the initial magnetic field $B_{0}$.

The results from these experiments are shown in figure 6.10-12 (a) and (b). The a-figures show the TOF distribution (circles) and the cooling spectra (triangles), 


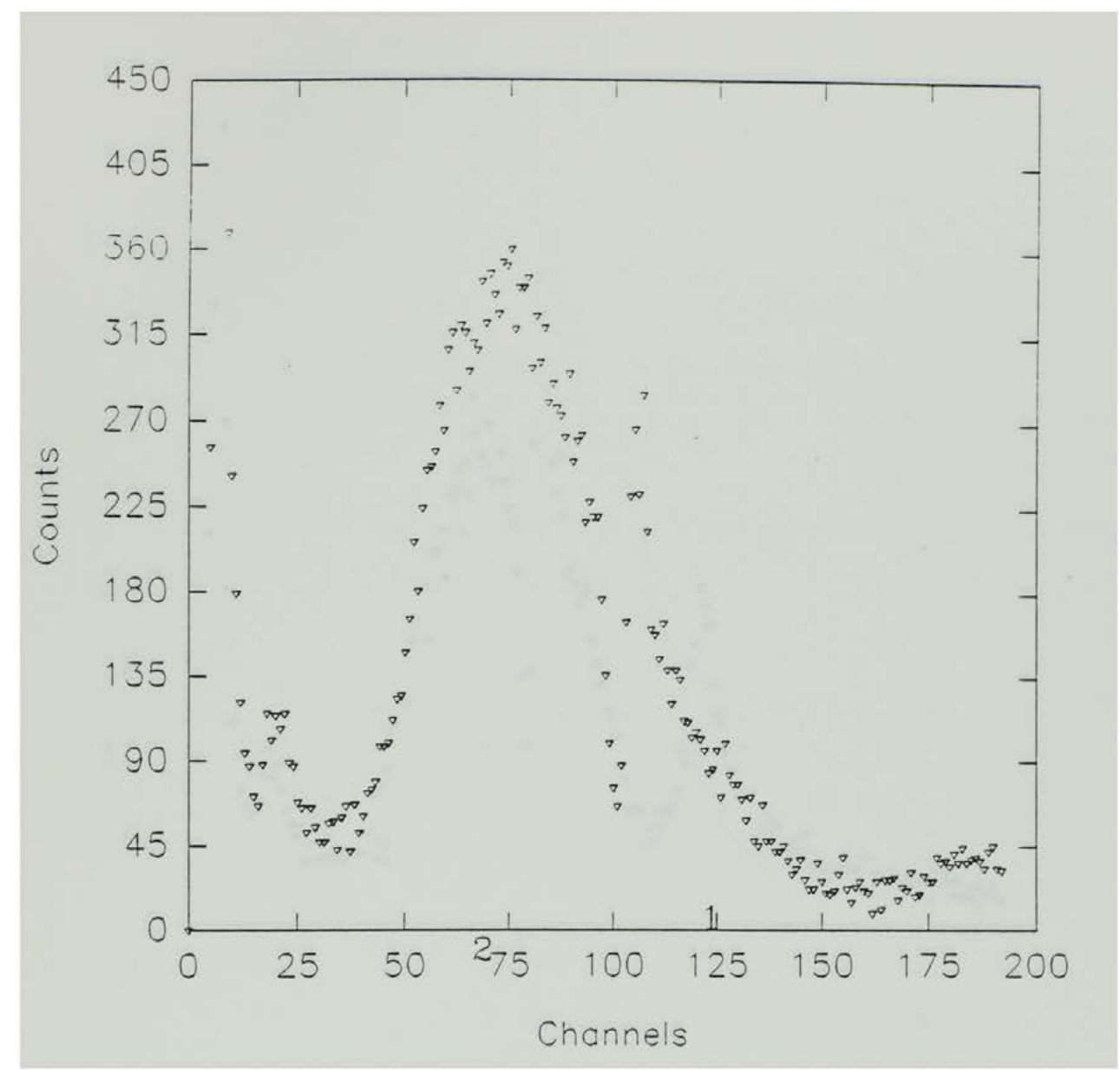

fig 6.7. Laser detuning is $351 \mathrm{Mhz}$, hole by the laser_1, magnetic field magnitude is 150 Gauss, hole by magnetic field: $\sigma^{+}{ }^{+}$.. 


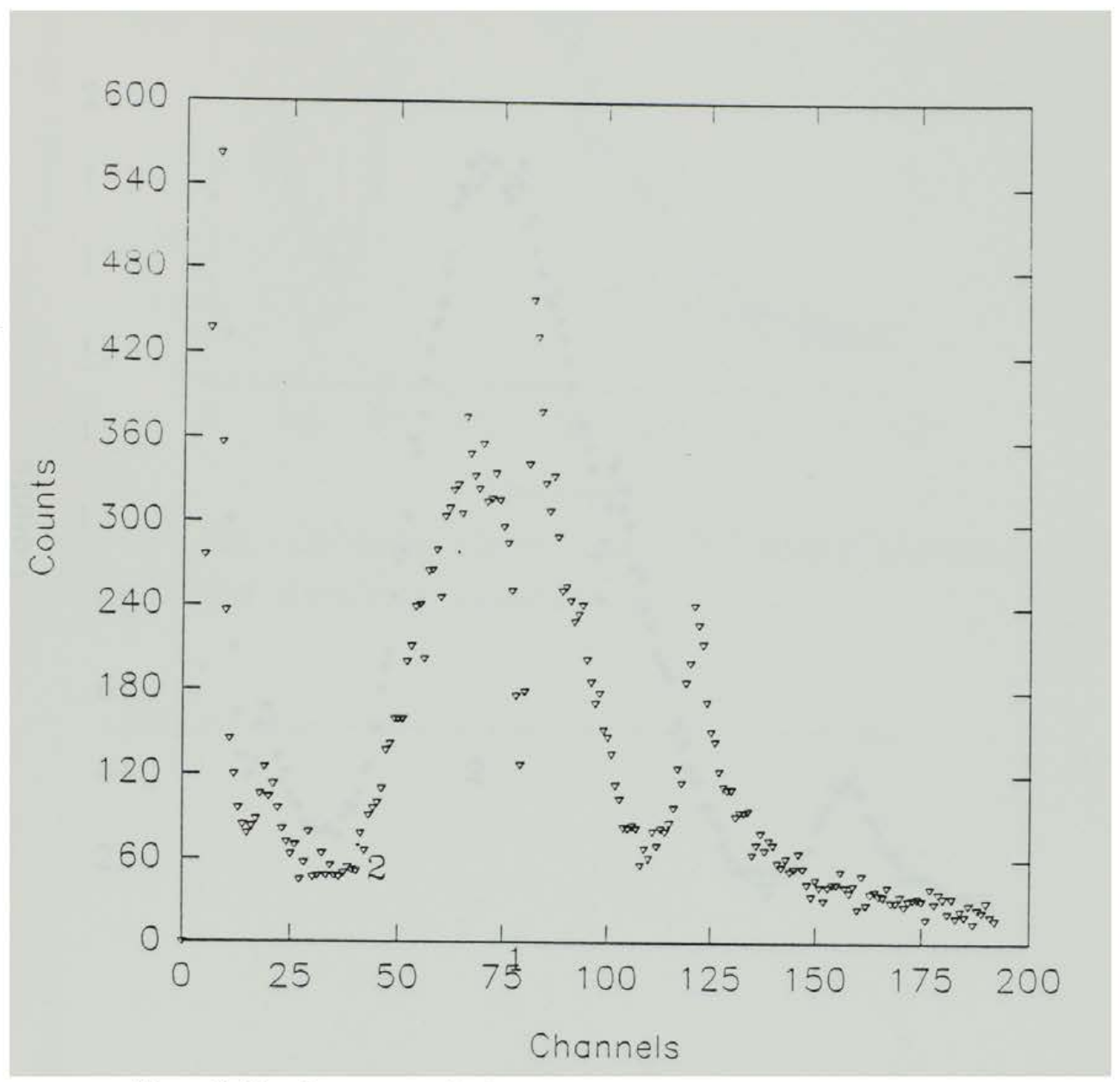

fig 6.8. Laser detuning is $520 \mathrm{Mhz}$, hole by the laser_1, magnetic field magnitude is 190 Gauss, hole by magnetic field $\sigma^{+} \_2$. 


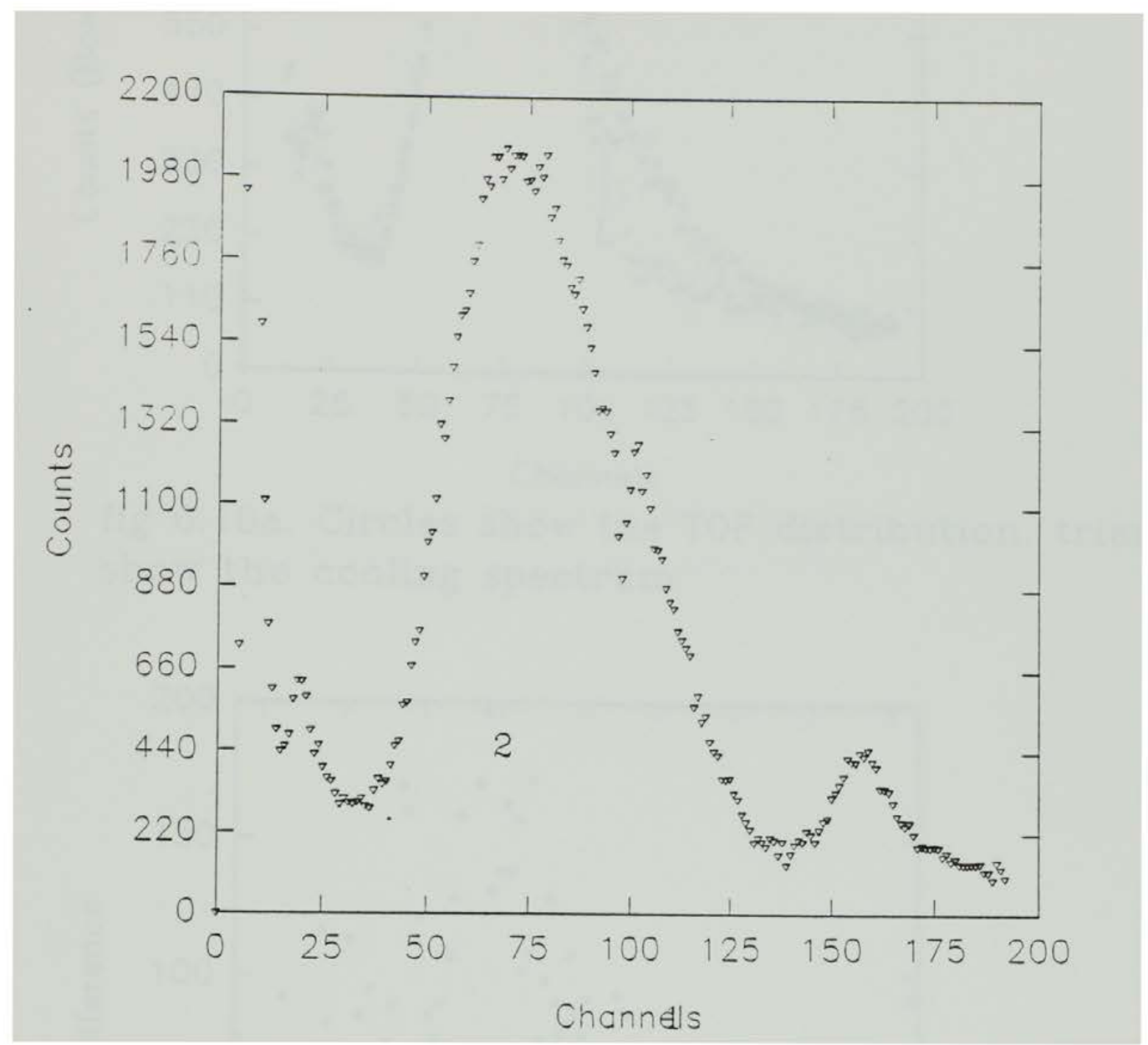

fig 6.9. Laser detuning is $408 \mathrm{Mhz}$, hole by the laser_1, magnetic field magnitude is 120 Gauuss, hole by magnetic field: $\sigma^{+}{ }^{+}$. 


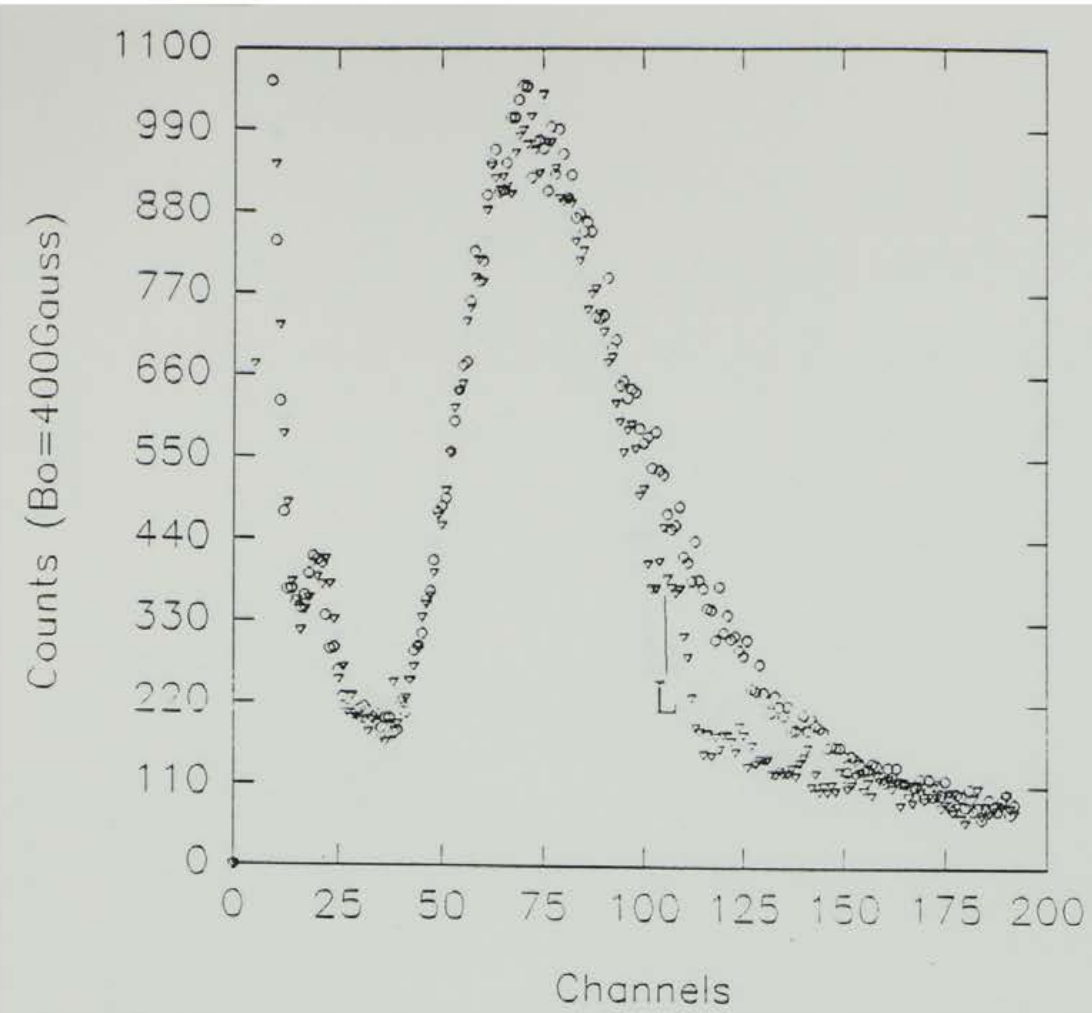

fig $6.10 \mathrm{a}$. Circles show the TOF distribution, triangles show the cooling spectrum

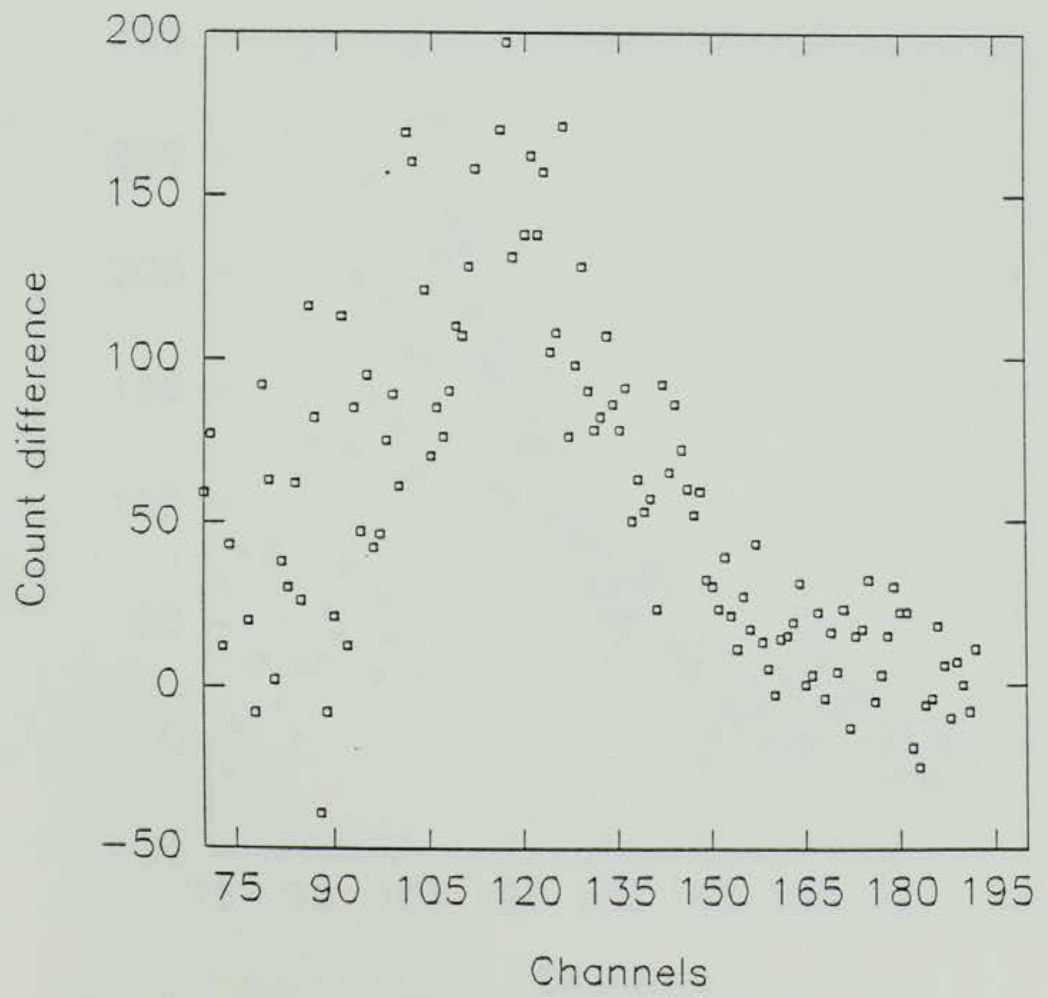

fig $610 \mathrm{~b}$. The absorption curve. 


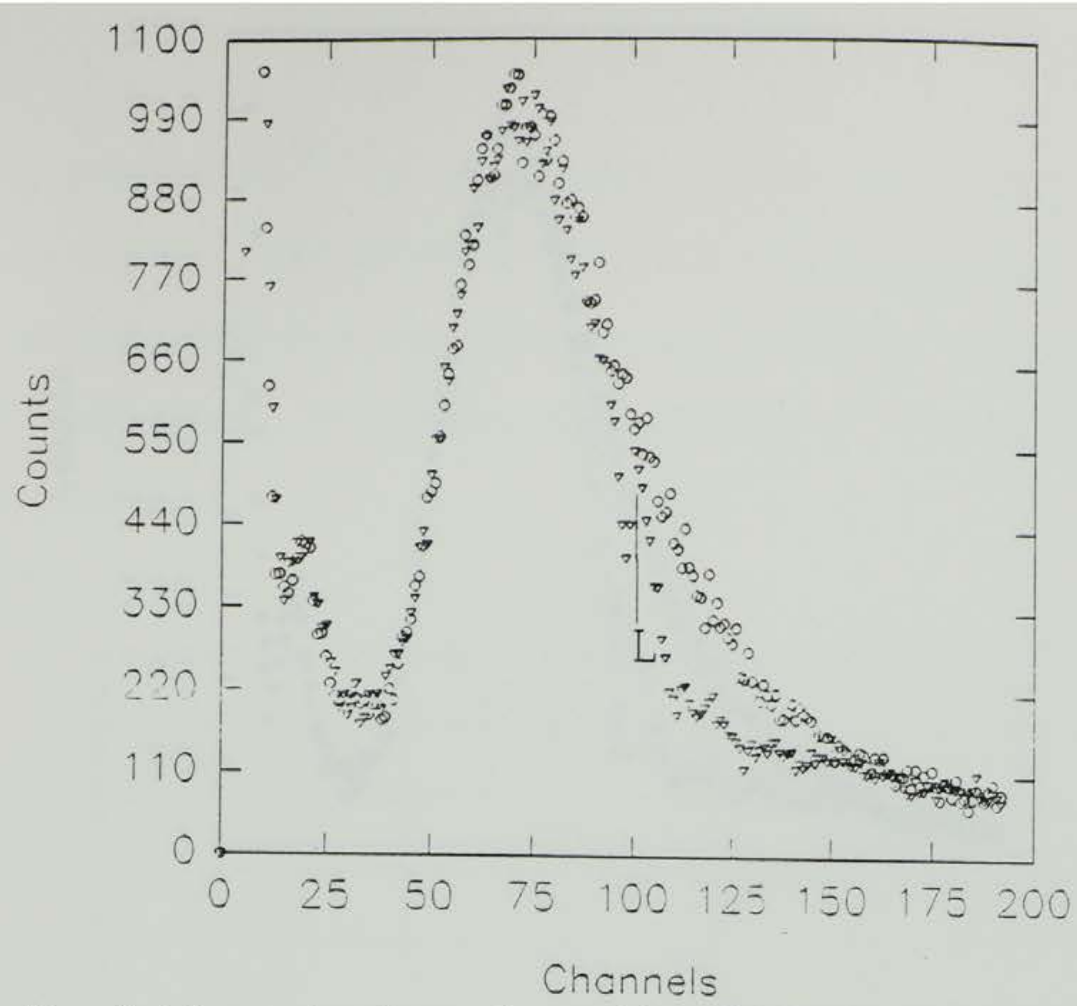

fig $6.11 \mathrm{a}$. circles show the TOF distibution, triangles show the cooling spectrum.

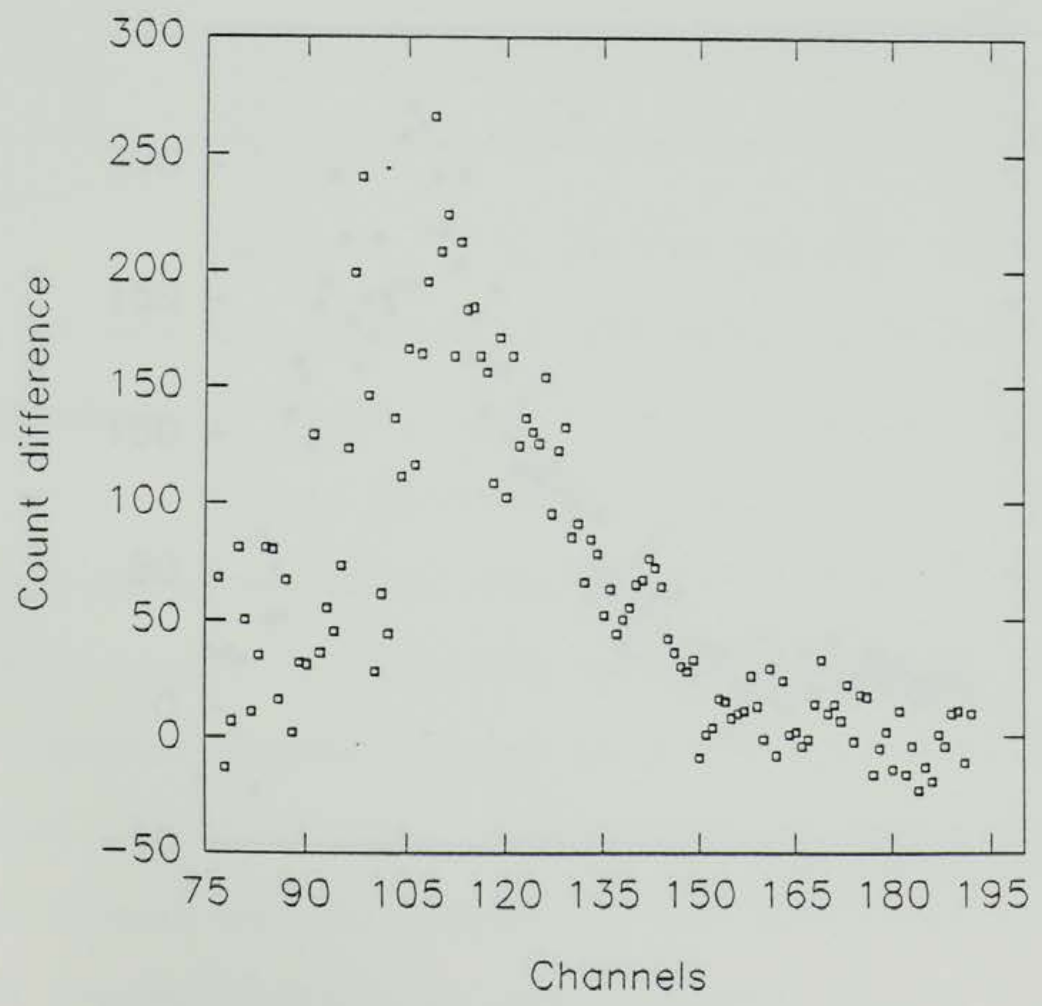

fig $6.11 \mathrm{~b}$. The absorption curve. 


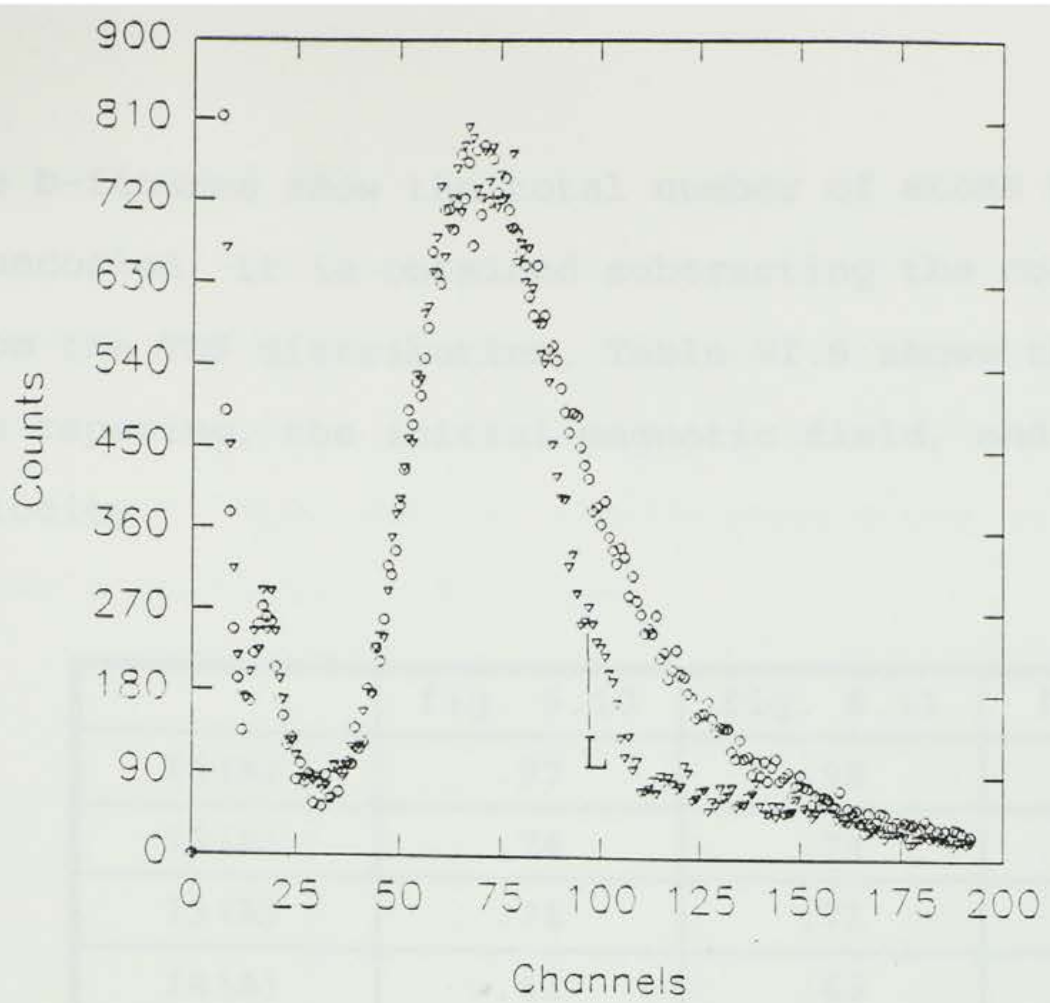

fig 6.12a. Circles show yht TOF distribution, triangles show the cooling spectrum.

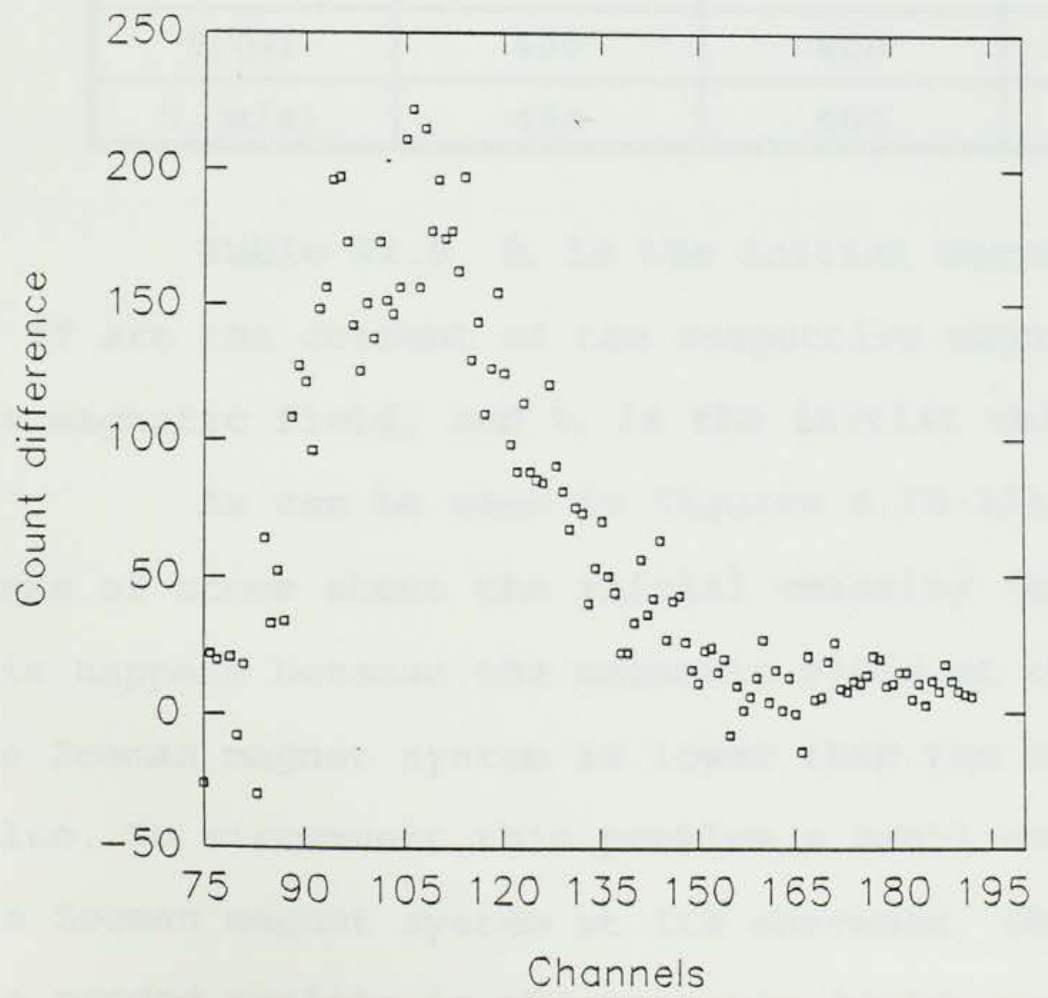

fig $6.12 \mathrm{~b}$. The absorption curve. 
the b-figures show the total number of atoms that have beencooled, it is obtained subtracting the cooling spectra from the TOF distribution. Table VI.5 shows the currents for the tapering, the initial magnetic field, and the initial velocity.

\begin{tabular}{||c|c|c|c||}
\hline & fig. 6.10 & fig. 6.11 & fig 6.12 \\
\hline I1 (A) & .97 & .98 & 1.22 \\
\hline I2 (A) & .74 & .74 & .9 \\
\hline I3 (A) & .71 & .71 & .87 \\
\hline I4 (A) & .63 & .63 & .74 \\
\hline I5 (A) & .56 & .56 & .65 \\
\hline I6 (A) & .43 & .43 & .52 \\
\hline I7 (A) & .37 & .37 & .43 \\
\hline$B_{0}(\mathrm{G})$ & 400 & 400 & 490 \\
\hline$V_{0}(\mathrm{~m} / \mathrm{s})$ & 454 & 454 & 556 \\
\hline
\end{tabular}

Table VI.5. $B_{0}$ is the initial magnetic field, II to I7 are the current of the respective magnets to tapered the magnetic field, and $v_{0}$ is the initial velocity.

As can be seen in figures 6.10-12a, there are leaks of atoms about the initial velocity (shown with an $L$ ), this happens because the magnetic field at the entrance of the Zeeman magnet system is lower than the theoretical value. To circumvent this problem a small coil was added to the Zeeman magnet system at its entrance, this magnet gives the needed profile in the magnetic field, such that, the magnetic field matches the theoretical field at the entrance 
of the system.

Since the next and most important step in these experiments is the trapping of neutral atoms, a larger number of atoms at zero or nearly zero velocity are needed. It is very important to involve many atoms in the cooling, atoms with higher velocities.

\begin{tabular}{||c|c|c||}
\hline$L(m)$ & $V_{0}(\mathrm{~m} / \mathrm{s})$ & $C h$ \\
\hline .85 & 606 & 75 \\
\hline .95 & 641 & 71 \\
\hline 1.05 & 673 & 68 \\
\hline 1.15 & 705 & 65 \\
\hline 1.25 & 735 & 62 \\
\hline
\end{tabular}

Table VI.6. L is the length of the magnet system, $\mathrm{Ch}$ is the channel on the TOF corresponding to $\mathrm{V}_{0}$ the velocity at which the cooling process could start. 


\section{CHAPTER VII - CONCLUSIONS}

In this thesis, the laser cooling of an Argon metastable atomic beam was successfully demonstrated using the momentum transfer from a counterpropagating laser beam and the Zeeman cooling approach to compensate for the changing Doppler shift that takes the atoms out of resonance during the cooling process. The magneto-optical trap (MOT) was built, but not tested due to laser problems. The atomic velocity distribution was determined by the TOF spectroscopy. This technique depends on the absolute measurement of the time of flight of the atoms along a known path; and for low velocities, gives a velocity resolution of a few times better than other techniques used (the natural width of the atomic cycling transition in the Argon $\gamma=1 / 2 \pi \tau \sim 5.6 \mathrm{MHz}$ gives a velocity resolution $\Delta \mathrm{v}=\lambda \gamma \sim 4.5 \mathrm{~m} / \mathrm{s}$ by this method).

The necessity of ultrahigh resolution spectroscopy was the motivation for the laser cooling, and the study and construction of the MOT. A future step in this work is the testing and improvement of the MOT for trapping neutral Argon atoms. The main task doing so will be to cool more atoms (1) and the alignment of the optical molasses with the trapping magnetic field (2).

The solution to (1) should be the use of a longer spatially varying field, which will allow a larger velocity 
reduction while keeping the rate of change of the field small enough that the atoms can stay in resonance as they decelerate. This will produce more atoms with final velocity very close to zero velocity, and a compression of the velocity distribution of the cooled atoms. The production of a very slow, cold atomic beam, with a velocity spread less than $6 \%$ and a high density of atoms, demonstrates the utility of laser deceleration for atomic-beam "velocity selection". The advantage of this laser velocity selection over mechanical selection is that unwanted velocities are compressed into the desired velocity rather than being discarded.

We are continuing to work on perfecting the magnetic profile for the Zeeman magnet system to eliminate the leakage of atoms from the cooling process. An small solenoid was built, tested and installed at the beginning of the Zeeman system, this solenoid gives the magnetic field needed to match the theoretical magnetic profile with the experimental one. This together with a larger Zeeman system should give all the cooled atoms needed for a successful trapping of Argon metastables, thus, the possibility of doing high resolution spectroscopy on Argon atoms. 


\section{LIST OF REFERENCES}

1. D.J.Wineland and H.Dehmelt, Bull. Am. Phys. Soc., 20, 637 (1975) .

2. J.C.Bergquist, D.J.Wineland, et al., phys. Rev. Lett. 55, 1567 (1985).

3. D.J.Larson, J.C.Bergquist, D.J.Wineland, et al., Phys. Rev. Lett. 57, 70 (1986).

4. J. Michael Hollas, High Resolution Spectroscopy, Butterworths, 8.2.8 Saturation Spectroscopy.

5. A. Einstein, Sitzungsber.Kgl.Preuss.Akad.Wiss.1924 261 (1924).

6. Bordé, C.J. Compt. rend.,271B, 371 (1970).

7. Smith, P.W. and Hänsch, T.W. Phys. Rev. Lett.,26,29 (1971).

8. Sorem,M.S. and Schawlow,A.L. (1972). Optics Commun., 5, 148

9. Wieman, C. and Hänsch, T.w. (1976) Phys.Rev.Lett. , 36,1170.

10. P.C.Engelking, Rev.Sci.Instrum.57, 2274 (1986)

11. Avila, C., Master Thesis, Florida International University (1996)

12. S.N.Bose, Z. Phys.26, 178 (1924).

13. M.H.Anderson, J.R.Ensher, M.R.Matthews, C.E.Wieman, E.A.Cornell. Science, Vol. 269, 14 July 1995.

14. C.C.Bradley, C.A.Sackett, J.J.Tollet, and R.G. Hulet. Phys. Rev. Lett., Vol 75, 9, 1687, 28 August 1995.

15. K.B.Davis, M.O. Mewes,M.R. Andrews, N.J. van Druten, W. Ketterle, et al., Phys.Rev.Lett., Vol 75, 22,3969, 27 Nov 1995. 
16. A. Einstein, Phys. Z. 18, 121 (1917). English

translation in Source of Quantum Mechanics, B.L. Van der Waerden (Ed.), (North Holland, Amsterdam, 1969).

17. P.W.Milonni, J.H.Eberly, Lasers, John Wiley \& Sons, 1988. 18. V.S.Letokhov, V.G.Minogin et al., Opt.Commun., 19, 72 (1976) .

19. Harold Metcalf, Atomic Cooling And Trapping. Encyclopedia of Applied Physics, Vol.2, 1991 VCH Publishers, Inc.

20. Pritchard D.E., Raab E.L., Bagnato D.S.,Wieman C.E., and watts R.N., (1986) Phys. Rev. Lett. 57, 310.

21. Dalibard, J., Laboratoire de Spectroscopie, Universite Paris VI.

22. Metcalf, H. (1989) J. Opt. Soc. Am. B Vol 6, No. 11, pp $2206-2210$

23. Coherent 899-21, Operator's Manual.

24. Second Interim Technical Report. Federal Demostration, Project F49620-93-1-0159DEF. FIU, Miami, Fl, 15 March 1995. 25. A. Barrios, et al., J. Appl. Phys.,76(2), 15 July 1994. 26. G.B.Ramos, et al., Phys. Review A, Vol 51, 4, April. 1995. 27. K.Hardy and J.W.Sheldon, Rev.Sci.Instrum., 52, 1802 (1981). 28. K.A. Hardy, et al., J. Appl. Phys., 67, 7240 (1990). 29. A. Barrios, K.A. Hardy, J.W. Sheldon and J.R. Peterson, Phys. Rev. Lett. 69, 1348 (1992). 
Appendix.1. Important Ar Characteristic to Cooling and Trapping .
Argon Mass (kg)
$6.64 * 10^{-26}$
Metastable level (eV)
$1 S_{5} \quad(11.5471)$
Lifetime (sec)
$>1.3$
Pump level (eV)
$2 \mathrm{P}_{9} \quad(13.074)$
Lifetime $(\tau)$ (sec)
$2.85 * 10^{-8}$
Transition wavelength $(\lambda)(m)$
$811.73 * 10^{-9}$
Resonance Frequency ( $\mathrm{MHz}$ )
$3.696 * 10^{5}$
Natural bandwidth ( $\mathrm{GHz})$
$(\gamma=1 / 2 \pi \tau)$
5.6

Saturate Intensity $\left(\mathrm{mW} / \mathrm{cm}^{2}\right)$

$$
\left(I_{\text {sat }}=2 \pi \mathrm{hc} / \tau \lambda^{3}\right)
$$

8.2

Maximum Acceleration (m/ $\left.\sec ^{2}\right)$

$$
\left(\mathrm{a}_{\max }=\mathrm{h} / 2 \tau \lambda \mathrm{M}\right)
$$

$2.16 * 10^{5}$

Most probable velocity $\left(\mathrm{V}_{\mathrm{p}}\right)(\mathrm{m} / \mathrm{sec}) \quad 700$

Doppler shift at $\mathrm{V}_{\mathrm{p}}$ (MHz)

$$
\left(v_{D}=v_{r} v_{p} / c\right)
$$

Distance to Stop (m)

$$
\left(z=V_{p}^{2} / 2 a_{\max }\right)
$$

Velocity Change per Photon (m/sec)

$$
(\triangle \mathrm{V}=\mathrm{h} / \lambda \mathrm{M})
$$

0.012

Number of Photons to Stop

$5.83 * 10^{4}$

Doppler Temperature $(\mu \mathrm{K})$

$$
\left(\mathrm{T}_{\mathrm{D}}=\hbar \gamma / 2 \mathrm{k}_{\mathrm{B}}\right)
$$


Minimal Damping Time (sec)

$$
\left(\tau_{\mathrm{c}}=2 \mathrm{M} / \hbar \mathrm{k}^{2}\right)
$$

$2 \cdot 1 * 10^{-5}$

Recoil Temperature $(\mu \mathrm{K})$

$$
\left(T_{c}=\hbar / \tau_{c} k_{B}\right)
$$

.33 